\title{
Modelling mixed mode fracture of mortar joints in masonry buildings
}

\author{
M. Bisoffi-Sauve, ${ }^{\mathrm{a}, *}$, S. Morel ${ }^{\mathrm{a}}$, F. Dubois ${ }^{\mathrm{b}}$ \\ ${ }^{a}$ Université de Bordeaux, UMR 5295 CNRS, Institut de Mécanique et d'Ingénierie - Bordeaux (I2M), Dépt. Génie Civil et Environnemental (GCE), Bordeaux F-33000, \\ France \\ ${ }^{\mathrm{b}}$ LMGC, CNRS, Univ. Montpellier, Montpellier, France
}

Keywords:

Discrete Element Method

Cohesive contact law

Masonry

Mortar joints

\begin{abstract}
A B S T R A C T
Within the framework of Discrete Element Modelling of masonry structures, a contact law based on Cohesive Zone Model and Coulomb's law is proposed to describe the fracture behaviour of mortar joints. The contact law is expressed in mixed mode I + II and is based on the cohesive behaviours of pure modes I and II and on the frictional behaviour whose parameters can be estimated from an experimental procedure consisting in two fracture tests. Moreover, it is shown from numerical simulations that the contact law exhibits a load path dependency in agreement with the quasi-brittle behaviour expected for mortar. Finally, a parametric study performed from the simulation of the diagonal compression of a masonry panel shows a good agreement of the obtained load-displacement responses and failure modes compared to experimental results of the literature.
\end{abstract}

\section{Introduction}

Masonry is one of the oldest building material and is thus widely present around us. But despite the simplicity of its manufacturing, it exhibits a complex behaviour, mainly influenced by the heterogeneity and anisotropy induced by the units arrangement and by the difference of material properties between the elements composing the structure. However, a better understanding of masonry is relevant for design optimisation of modern structures, but also for the conservation of ancient buildings that are part of our heritage.

Many researchers proposed different approaches for the numerical study of masonry structures, depending on the level of accuracy and the simplicity desired. Macro-modelling considers the structure as a homogenised continuum, averaging stresses and strains at global level. Some macro-models are derived from concrete approach like smeared crack models $[26,28,15]$ but such models are more adapted to grouted masonry because they do not take into account anisotropy due to mortar joints [2]. Other macro-models were developed especially for masonry and failure criteria are derived directly from experimental data $[21,16]$. These approaches can give good idea of the behaviour of the structure but are limited to conditions under which experimental data are obtained.

As regards to multi-scale models, homogenisation techniques $[16,18]$ consider masonry as a periodical arrangement. This periodicity allows to consider the media as a repetition of elementary cells that take into account the behaviour of its constitutive materials. Stresses and strains of the equivalent homogenised media are expressed from the description of the elementary cell. The structure behaviour is thus deduced from the properties of local elements.

To better understand masonry at a local level and take real geometry into account, several micro-models have been proposed $[16,27,25]$. They represent blocks, mortar joints and block/mortar interfaces separately and allow to observe mechanisms at block level, joint level, or combined mechanisms of block and joint. These approaches can represent local damage and global behaviour of masonry structures but are very time consuming because of the large number of represented elements. Consequently, these methods are not well adapted to large scale structures. To tackle this limitation, simplified micro-models are possible $[16,12,6,29,30]$, where mortar joints and block/mortar interfaces are lumped into one interface element. In this framework, Discrete Element Methods (DEM) [7,19] allows both microand simplified micro-modelling. Indeed, DEM describe collections of bodies (rigid or deformable) in interaction and global behaviour is deduced from local behaviour. Hence, this method is well suited for the modelling of a discontinuous media such as masonry.

The aim of this work is to develop an accurate model, able to describe the fracture behaviour at the scale of mortar joints, i.e. at a mesoscopic scale. Within the framework of Discrete Element Method, the progressive damage of mortar joints and the frictional behaviour of fractured joints are described with a Cohesive Zone Model (expressed in mixed-mode I + II) and with Coulomb's law respectively.

Firstly, we briefly introduce Non Smooth Contact Dynamics method

\footnotetext{
* Corresponding author.

E-mail address: marie.sauve@ymail.com (M. Bisoffi-Sauve).
} 
(NSCD) that will be used for discrete modelling with LMGC90 software. Then, we present the cohesive interface law that was implemented in order to model progressive damage of mortar joints. As we will see, this formulation is based on cohesive parameters corresponding to joints fracture in pure Mode I (opening) and pure Mode II (sliding), which can be easily estimated from two experimental tests.

\section{Discrete modelling with non smooth contact dynamics}

Discrete element models have the ability to represent collection of bodies in interaction and allow to take into account the discontinuous nature of a media. The structure is described at a local scale through interaction laws, and the global behaviour is then deduced from local behaviour.

Generally, discrete element methods rely on few hypotheses [9]:

(i) Interaction depends only on the properties of the two bodies in contact,

(ii) Contact region is small compared to the element size: contact can be considered as punctual,

(iii) Strain is still confined to the contact region, so bodies can be considered as rigid,

(iv) Interactions are independent from each other so that the contact force can be determined from the strain at the contact region.

Contact Dynamics was first achieved by Moreau [19] and allows to simultaneously deal with all active interactions over a time step, ignoring the 4th hypothesis (iv). Later, Jean [14] extends this method and adapts it to collections of deformable bodies, giving birth to the so called Non Smooth Contact Dynamics (NSCD) that neglects the hypothesis (iii). The method uses an implicit time integrator and "Non Smooth" refers to the use of interaction laws not necessarily regular. The NSCD is implemented into the LMGC90 software $[8,10]$, that will be used for discrete modelling.

Over a time step, three important steps are necessary to solve the problem (Fig. 1):

- Contact detection

- Computation of contact forces, called contact problem

- Determination of bodies motion

Indeed, two sets of unknowns appear in the multi-contact problem: local unknowns related to the interactions, and global unknowns related to the bodies (Fig. 2). Mappings exist between these two levels. For a contact $\alpha$, the operator $\mathbf{H}^{\alpha}$ gives the contribution $\mathbf{R}^{\alpha}$ to global resultant forces due to local forces $\mathbf{R}^{\alpha}$ :

$\mathbf{R}^{\alpha}=\mathbf{H}^{\alpha}(q) \mathcal{R}^{\alpha}$

where $q$ represents the vector of generalised degrees of freedom.

Using duality consideration, velocity relative to contact can also be related to body velocity with transposed operator $\mathbf{H}^{T}$ :

$\mathcal{V}^{\alpha}=\mathbf{H}^{T \alpha}(q) \mathbf{V}^{\alpha}$

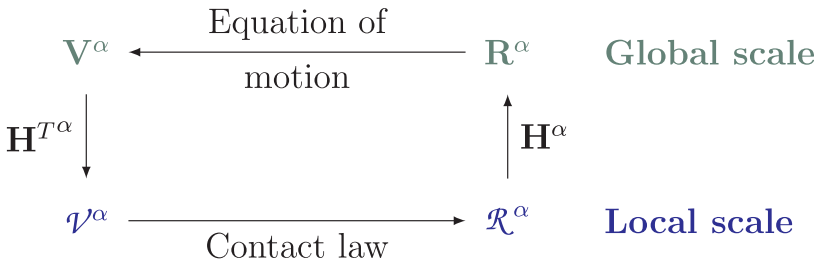

Fig. 2. Local/global mapping.

For each contact, the relative velocity and reaction force are related through a condition of Signorini-Coulomb. The unilateral Signorini condition (Fig. 3a) describes the impenetrability between two bodies and is written as:

$\left\{\begin{array}{l}g \geqslant 0 \\ \mathcal{R}_{n} \geqslant 0 \\ \text { g. } \mathcal{R}_{n}=0\end{array}\right.$

where $g$ is the gap between two bodies and $\mathcal{R}_{n}$ the normal contact force. For dynamical issues, it is more natural to formulate the above system in terms of velocities:

$\left\{\begin{array}{l}g\left(t_{0}\right) \geqslant 0 \text { at initial step } t_{0} \\ g(t) \leqslant 0 \Rightarrow \mathcal{V}_{n} \geqslant 0, \mathcal{R}_{n} \geqslant 0, \mathcal{V}_{n} \mathcal{R}_{n}=0\end{array}\right.$

where $\mathcal{V}_{n}$ is the normal component of velocity relative to the contact.

The Coulomb's law (Fig. 3b) allows to express shear sliding resistance between two bodies in contact and is written as:

$\begin{cases}\left\|\mathcal{R}_{t}\right\|<\mu \mathcal{R}_{n} \Rightarrow\left\|\mathcal{V}_{t}\right\|=0 & \text { Sticking } \\ \left\|\mathcal{R}_{t}\right\|=\mu \mathcal{R}_{n} \Rightarrow\left\|\mathcal{V}_{t}\right\|=-\lambda \frac{\mathcal{R}_{t}}{\left\|\mathcal{R}_{t}\right\|} & \text { Sliding }\end{cases}$

with $\lambda>0$ and $\mu$ the friction coefficient.

Usually the motion of each body composing the divided media can be described by the dynamics equation:

$\mathbf{M}(q) \dot{\mathbf{V}}=\mathbb{F}_{\text {int }}(q, \mathbf{V}, t)+\mathbb{F}_{\text {ext }}(t)+\mathbf{R}$

+ Initial conditions and boundary conditions

where:

- $\mathbf{M}$ is the inertia matrix

- $\mathbb{F}_{\text {int }}$ refers to internal forces (deformable bodies) and the non-linear inertia terms (centrifugal and gyroscopic)

- $\mathbb{F}_{\text {ext }}$ represents the external forces

- $q$ corresponds to the vector of generalised degrees of freedom

- $\mathbf{V}$ is the velocity vector

- $\mathbf{R}$ represents contact forces

In order to manage collisions and non smooth events, dynamics (6) is reformulated in terms of differential measures Dubois et al. [10]. Then, considering a time-interval $\left.] t_{i}, t_{i+1}\right]$ of length $h$, the previous equation can be integrated as:

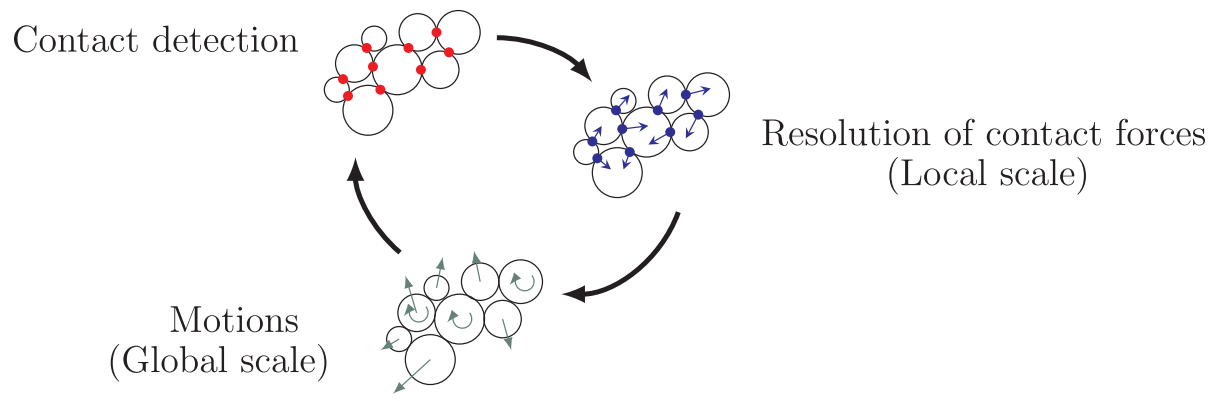

Fig. 1. Resolution of the contact problem. 


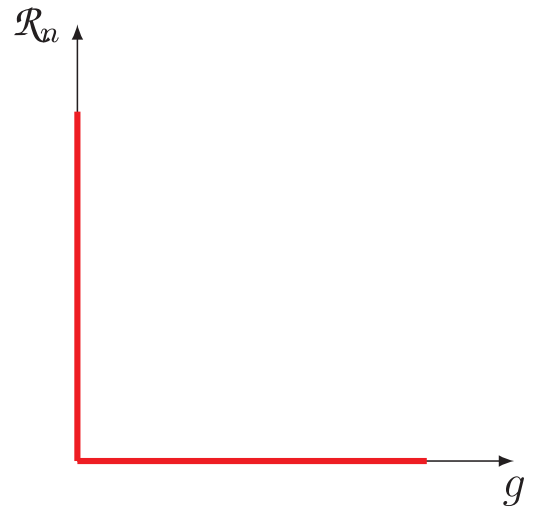

(a) Signorini condition

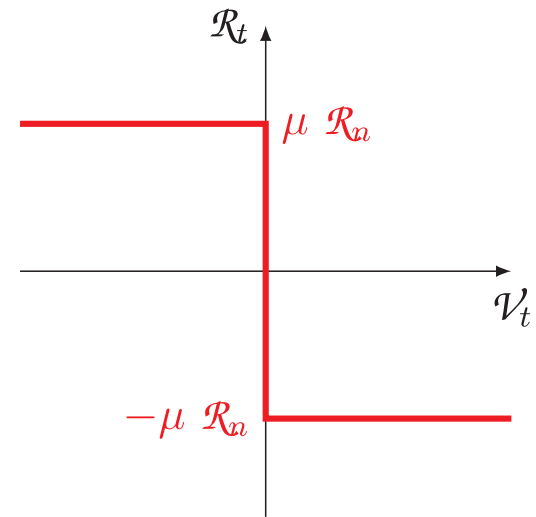

(b) Coulomb's law

Fig. 3. Unilateral contact conditions.

$\mathbf{M}\left(\mathbf{V}_{i+1}-\mathbf{V}_{i}\right)=\int_{t_{i}}^{t_{i+1}} \mathbb{F}_{\text {ext }}+\mathbb{F}_{i n t} \mathrm{~d} t+\mathbf{I}$

where $\mathbf{I}$ is the impulse of contact resultant.

Or:

$\mathbf{V}_{i+1}=\underbrace{\mathbf{V}_{i}+\mathbf{M}^{-1} \int_{t_{i}}^{t_{i+1}} \mathbb{F}_{\text {ext }}+\mathbb{F}_{\text {int }} \mathrm{d} t}_{\mathbf{V}_{\text {free }}}+\mathbf{M}^{-1} \mathbf{I}$

The term $\mathbf{V}_{\text {free }}$ represents the free velocity, i.e. the velocity the body would have without any contact.

The previous equation is then expressed with local unknowns, and using the Delassus operator $\left(\mathbb{W}^{\alpha \beta}=\mathbf{H}^{T \alpha}\left(q_{m}\right) \mathbf{M}^{-1} \mathbf{H}^{\beta}\left(q_{m}\right)\right)$, the problem can be sum up as:

$\left\{\mathcal{V}_{i+1}^{\alpha}=\mathcal{V}_{\text {loc,free }}^{\alpha}+\mathbb{W}^{\alpha \alpha} \mathcal{I}^{\alpha}\right.$

Contact law $\left(\mathcal{I}^{\alpha}, \mathcal{V}^{\alpha}\right)=0$

where $\mathcal{V}_{\text {loc,free }}^{\alpha}=\mathbf{H}^{T \alpha}\left(q_{m}\right) \mathbf{V}_{\text {free }}+\sum_{\beta \neq \alpha} \mathbf{W}^{\alpha \beta} \mathcal{I}^{\beta}$.

The resolution of the contact problem is done using a Non-Linear Gauss Seidel method Jean [14].

\section{Formulation of cohesive contact in mixed mode I + II}

\subsection{Cohesive contact law}

The contact forces calculation is based on the definition of contact laws at the interfaces. LMGC90 has been already successfully used for the modelling of masonry structures $[22,24,32]$, but mainly with frictional contact laws, or Mohr Coulomb criterion. However, a lot of masonry structures include mortar joints between stone blocks that confer cohesion at the contact level. The fracture behaviour of mortar joints is known to be quasi-brittle, meaning that the failure of the joint is preceded by a progressive damage resulting in a progressive decrease of normal and tangential stiffness. Such a behaviour can be represented by a Cohesive Zone Model, which describes the behaviour of a material through a constitutive law that relates the stress at the interface to the displacement discontinuity.

In this study, the structure, composed of masonry blocks linked by mortar joints, is modelled by lumping mortar joints and block/mortar interfaces into a unique interface (Fig. 4). This approach corresponds to the simplified micro-modelling of Lourenço [16] that we will be called thereafter mesoscopic approach.

Here, the constitutive law will admit an elastic part followed by a concave softening branch, typical of quasi-brittle materials. The softening part is here approximated by a bilinear behaviour (Fig. 5a), according to Morel et al. [20]. The bilinear softening branch is subdivided into two parts which are assumed to represent the two main mechanisms activated during quasi-brittle failure, namely micro-cracking and crack-bridging. The first part takes place after reaching the peak stress and until the cross-over displacement $\delta^{c}$ and mobilises micro-cracking energy $J^{\mu}$. In the second part, crack-bridging energy $J^{b}$ is consumed until the ultimate displacement $\delta^{u}$. After $\delta^{u}$, the interface is fully broken and no more cohesive stress is transmitted. Then, a classic Coulomb's friction law takes place.

Hence, the shape of the cohesive function is given by:

(i) the initial stiffness $K^{0}$

(ii) the maximal stress $\sigma^{e}$

(iii) the total cohesive energy $J^{c}$

(iv) the energy distribution between the two cohesive energies $J^{\mu}$ and $J^{b}$, defined by the ratio $\Phi=J^{\mu} / J^{c}$

(v) the ultimate opening $\delta^{u}$

The damage variable $d$, corresponding to the stiffness degradation ( $\left.d=K / K^{0}\right)$, evolves from 1 for an intact interface, to 0 for a fully broken interface.

For the shear behaviour of the interface (Mode II), in presence of normal compressive stresses, a friction threshold is considered (Fig. 5b). Its value is equal to $\mu . \sigma_{I}$, where $\mu$ corresponds to the friction coefficient and $\sigma_{I}$ to the normal compressive stress. In this configuration, the friction threshold has to be exceeded to activate the shear cohesive behaviour.

In the mixed mode formulation detailed thereafter, the mixed cohesive behaviour is expressed from the cohesive behaviours corresponding to pure modes of opening (Mode I) and sliding (Mode II). Thus, the parameters (i) to (v) given above will have to be defined for these two modes.

\subsection{Account for mixed mode}

Stresses at the interface between two blocks rarely correspond to pure mode solicitations, but are more likely combinations of these modes. Therefore material failure can be defined as a relationship between opening and sliding displacements and their corresponding normal and shear stresses at the interface level. The mixed mode is expressed thanks to two criteria that allow to couple pure modes under one mixed mode: one related to the damage initiation and the other to the failure (Fig. 6).

The damage initiation is expressed with a quadratic stress criterion. This criterion denotes the end of the elastic behaviour of the interface and is expressed as function of the pure modes maximal stresses. Considering that normal compressive stresses do not induce damage, we can write: 


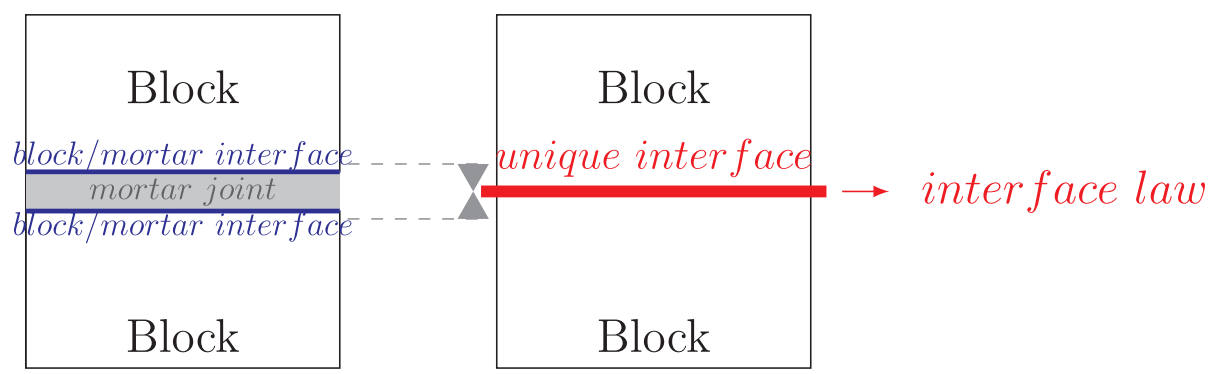

Fig. 4. Contact at the interface between blocks.

$\left(\frac{\sigma_{m I}^{e}}{\sigma_{I}^{e}}\right)^{2}+\left(\frac{\sigma_{m I I}^{e}}{\sigma_{I I}^{e}}\right)^{2}=1$

Furthermore, considering that initial stiffnesses of mixed mode components $\left(K_{m I}^{0}\right.$ and $\left.K_{m I I}^{0}\right)$ are equal to pure mode initial stiffnesses $\left(K_{I}^{0}\right.$ and $K_{I I}^{0}$ ), the above equation can be rewritten as function of relative displacements:

$\left(\frac{\delta_{m I}^{e}}{\delta_{I}^{e}}\right)^{2}+\left(\frac{\delta_{m I I}^{e}}{\delta_{I I}^{e}}\right)^{2}=1$

Then, defining a mixed mode displacement in the space of normal and tangential displacements:

$\delta_{m}=\sqrt{\delta_{m I}^{2}+\delta_{m I I}^{2}}$,

and a displacement ratio $\beta$ representing the participation of each mode:

$\beta=\frac{\delta_{m I I}}{\delta_{m I}}$,

we can finally express the displacement corresponding to damage initiation in mixed mode as function of $\beta$ and of pure modes displacements:

$\delta_{m}^{e}=\delta_{I}^{e} \cdot \delta_{I I}^{e} \cdot \sqrt{\frac{1+\beta^{2}}{\delta_{I I}^{e}+\delta_{I}^{e} \cdot \beta^{2}}}$

The second criterion describes the failure of the contact law in mixed mode. It is expressed from the pure modes fracture energies $J_{I}^{c}$ and $J_{I I}^{c}$ through the relationship:

$\left(\frac{J_{m I}}{J_{I}^{c}}\right)^{n}+\left(\frac{J_{m I I}}{J_{I I}^{c}}\right)^{m}=1$

where $\mathrm{n}=\mathrm{m}=1$ is considered in a first approximation.

Energy distribution $(\Phi)$ is assumed to be the same for pure traction and shear, as well as for mixed mode. Indeed, it seems reasonable to assume that micro-cracking and crack-bridging end at the same time for any direction. This leads to express the cross-over displacement corresponding to mixed-mode $\delta_{m}^{c}$ as function of the displacement ratio $\beta$ and pure modes displacements such as:

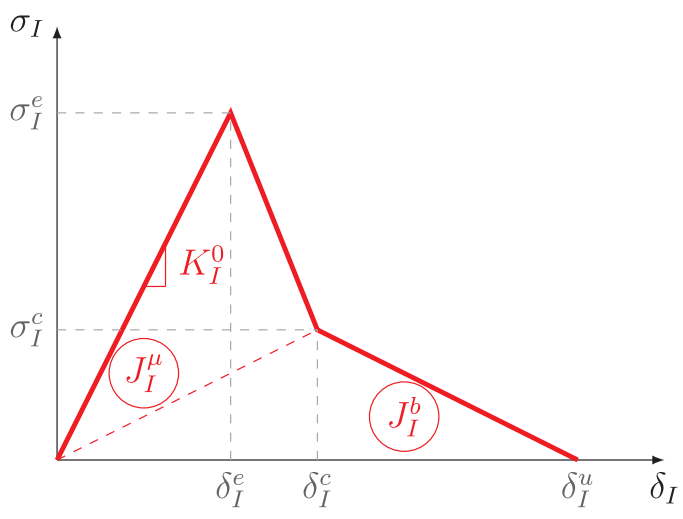

(a) Mode I cohesive law
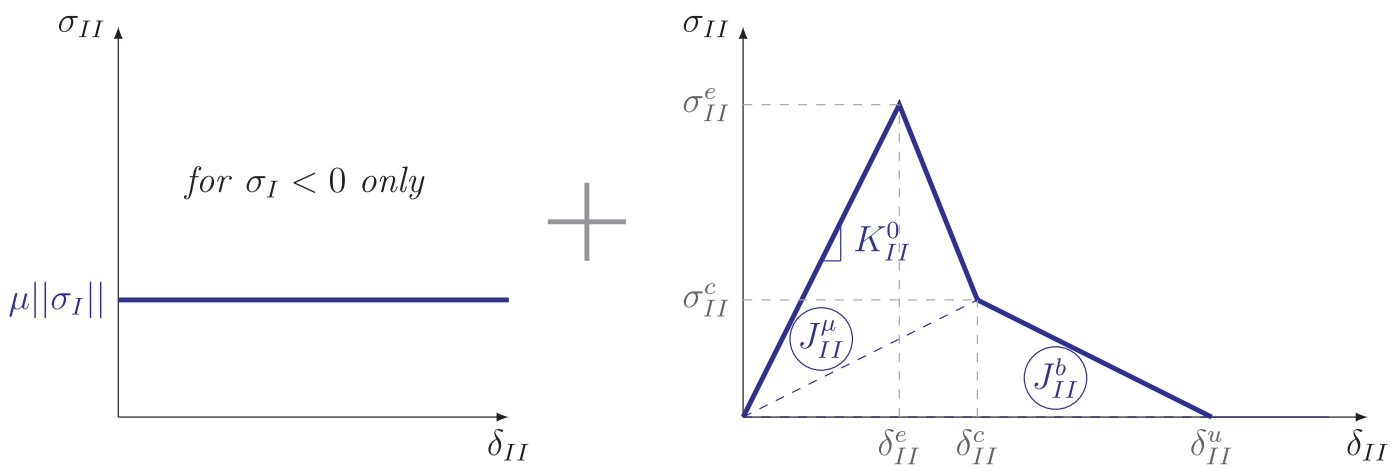

(b) Mode II cohesive law

Fig. 5. Pure Modes for the mixed-mode of cohesive law. 


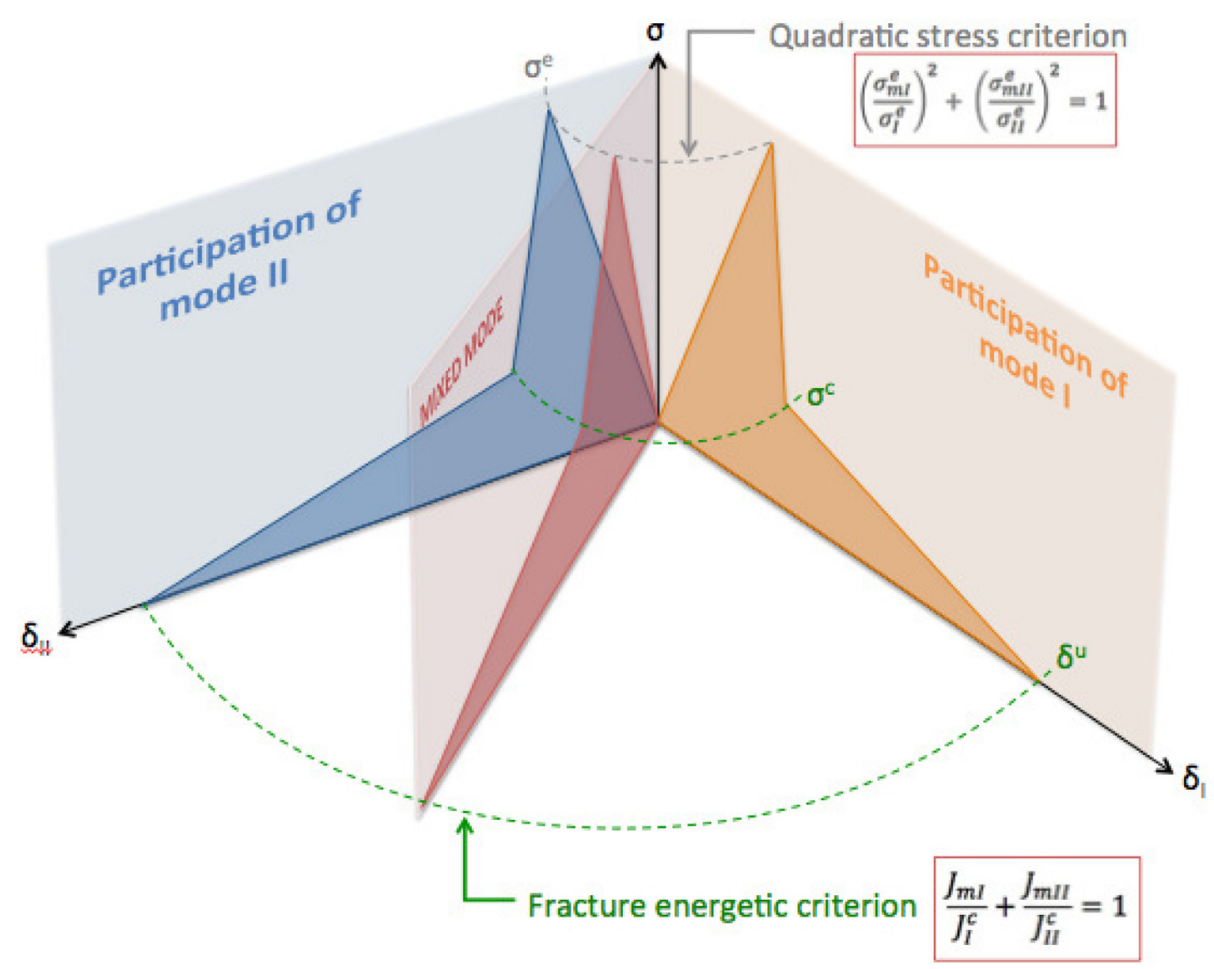

Fig. 6. Mixed mode contact law.

$\delta_{m}^{c}=\delta_{I}^{c} \delta_{I I}^{c} \sqrt{1+\beta^{2}} \frac{\sqrt{\delta_{I I}^{e}+\beta^{2} \delta_{I}^{e}}}{\delta_{I I}^{e} \delta_{I I}^{c}+\beta^{2} \delta_{I}^{e} \delta_{I}^{c}}$

On the basis on the same assumption as above, we can write the mixedmode ultimate displacement $\delta_{m}^{u}$ as:

$\delta_{m}^{u}=\frac{1+\beta^{2}}{\delta_{m}^{c}} \frac{\delta_{I}^{c} \delta_{I}^{u} \delta_{I I}^{c} \delta_{I I}^{u}}{\delta_{I I}^{c} \delta_{I I}^{u}+\beta^{2} \delta_{I}^{c} \delta_{I}^{u}}$

Mixed-mode is now defined from pure mode parameters, for any space configuration.

\section{Identification of cohesive and frictional parameters of mortar joints}

Mixed mode cohesive law is formulated from cohesive laws in pure Mode I (opening) and pure Mode II (sliding). The needed input parameters for the model are the initial stiffnesses $\left(K_{I}^{0}, K_{I I}^{0}\right)$, the maximal strengths $\left(\sigma_{I}^{e}, \sigma_{I I}^{e}\right)$, the cohesive fracture energies $\left(J_{I}^{c}, J_{I I}^{c}\right)$ and ultimate displacements $\left(\delta_{I}^{u}, \delta_{I I}^{u}\right)$ for both pure modes. The ratio $\Phi$ of microcracking energy over total cohesive energy and the friction coefficient $\mu$ are also needed.

These parameters can be estimated from characterisation tests carried on assemblies of sufficiently large size to ensure the observation of the mechanical behaviour at the mesoscopic scale. The cohesive parameters related to Mode I fracture of joints will be characterised from a direct tensile test performed on two blocks (one mortar joint), while the cohesive parameters related to Mode II fracture and friction coefficient will be estimated from a shear test on stone triplet.

All tested specimen in this study were constituted of limestone typical of the region of Bordeaux (France) and mortar was composed of sand, hydraulic lime and water (5:1:1). This type of block/mortar combination can be found in historical buildings of Bordeaux area.

\subsection{Mode I characterisation: direct tensile test}

\subsubsection{Experimental set-up}

Direct tensile tests are complicated to perform in a mechanical point of view, because the device have to induce pure traction to the specimen without any rotation. Therefore, the setting should not have any degree of freedom in order to generate uniform damage within the mortar joint [26]. Moreover, the test should minimise the size of the specimen for a more uniform crack propagation [13]. Some authors already realised direct tensile tests for the characterisation of masonry assemblies, but had difficulties to obtain post-peak behaviours of specimens $[23,4]$.

We propose a stiff set to prevent rotations (Fig. 7) where stone blocks are glued inside steel boxes. Blocks are approximately 2 centimetres higher than the boxes, so that force application is quite closed to the mortar joint and allow to install the metrology devices in the vicinity of the mortar joint. The bottom box is fixed while the traction force is applied through the upper box.

Displacements are measured with extensometers placed at the joint level in the four corners of the specimen where the most important displacements are expected. The test is displacement controlled with the average of the four extensometers displacements in order to estimate the softening behaviour of the joint. The displacement rate is set to $0.5 \mu \mathrm{m} / \mathrm{min}$. During the test, the tensile stress and the opening displacement of mortar joint are continuously recorded.

This set-up allows to obtain complete stress-displacement curves (elastic and softening parts) for $75 \%$ of the tests. These are improved results compared to other direct tensile tests carried on the same type of assembly $[23,4]$ that gave about $25 \%$ of usable data curves.

\subsubsection{Experimental results}

In most cases crack propagated at the interface between mortar joint and stone block. Twelve tests gave a usable stress-displacement curve (Fig. 8). As shown in Fig. 8, the scattering of the experimental results is large, but in the same order as the one obtained by Van der Pluijm on masonry assemblies submitted to direct traction [23]. This scattering can have different sources such as carving of stones and the irregularity of joint manufacturing from one specimen to one another. But above all, masonry assemblies are made from materials (stones and mortar) which themselves present a broad scattering by nature. Nevertheless, in spite of a large scattering, the softening regime emphasising the 

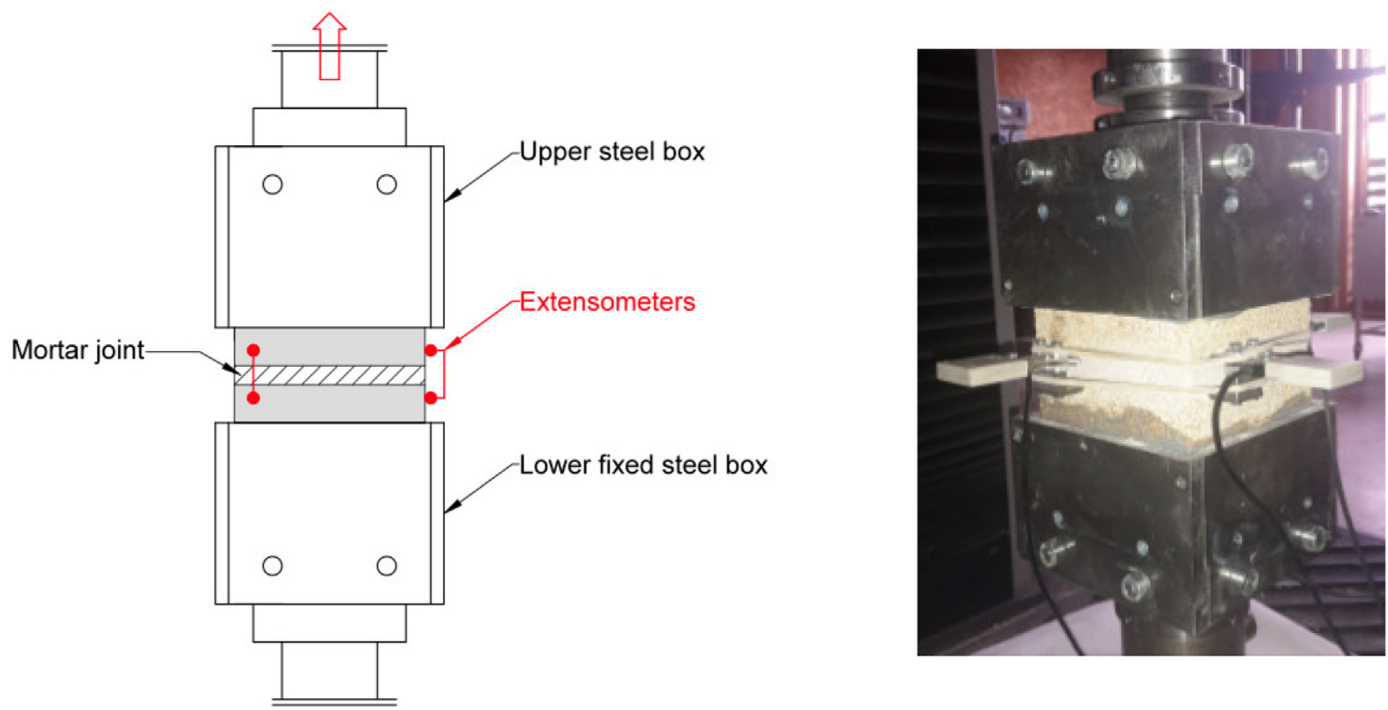

Fig. 7. Tensile test set-up.

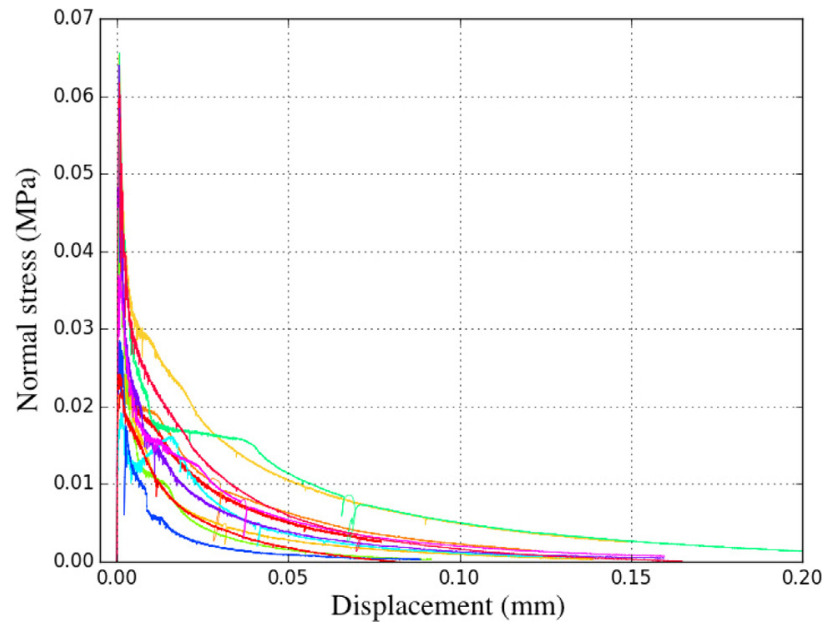

Fig. 8. Experimental curves from direct tensile tests.

progressive damage of mortar joint associated to joint opening is clearly observed from experimental response. As expected, this behaviour exhibits a concave shape and the curves allow a direct estimation of cohesive parameters:

- The initial stiffness is determined with a linear regression before the peak stress

- Maximal tensile stress and ultimate displacement are directly estimated on $\sigma-\delta$ response

- Cohesive fracture energy is estimated as the area under the $\sigma-\delta$ curve

The bilinear approximation of the softening behaviour can be estimated from the value of the cohesive energy and the value of the energy ratio $\Phi$ leading to the better fit of the concave shape of the softening.

The cohesive parameters found for Mode I for ashlar stones linked by lime mortar are given in Table 1 .

\subsection{Mode II characterisation: triplet shear test}

\subsubsection{Experimental set-up}

The triplet shear test is recommended by EN-1052-3 to characterise the initial shear resistance and is thus widely used. In the present study we propose a modification of the normalised set-up in order to observe
Table 1

Cohesive parameters obtained for Mode I.

\begin{tabular}{cc}
\hline \multicolumn{2}{c}{ Mode I } \\
\hline$\sigma_{I}^{e}(\mathrm{MPa})$ & $0.04(0.44)$ \\
$K_{I}^{0}(\mathrm{~Pa} / \mathrm{m})$ & $1.5 \times 10^{11}(0.60)$ \\
$J_{I}^{c}\left(\mathrm{~J} / \mathrm{m}^{2}\right)$ & $0.79(0.55)$ \\
$\delta_{I}^{u}(\mathrm{~mm})$ & $0.14(0.26)$ \\
\hline
\end{tabular}

the softening regime which leads to the estimation of the cohesive and frictional parameters of mortar joints.

The specimens are made of three ashlar limestone blocks linked by two mortar joints. The test is performed with a normal stress to the joints which facilitates the obtention of post-peak curve and allows to determine the frictional behaviour of the failed joints. Three compression levels were tested: $0.2 \mathrm{MPa}, 0.3 \mathrm{MPa}$ and $0.4 \mathrm{MPa}$.

The two external blocks are kept fixed while the shear force is applied to the central one. Test was displacement controlled, with the mean displacement of 4 LVDT placed on the two sides of the specimen, near the two joints (Fig. 9). The displacement rate is set to $0.06 \mathrm{~mm} /$ min before peak.
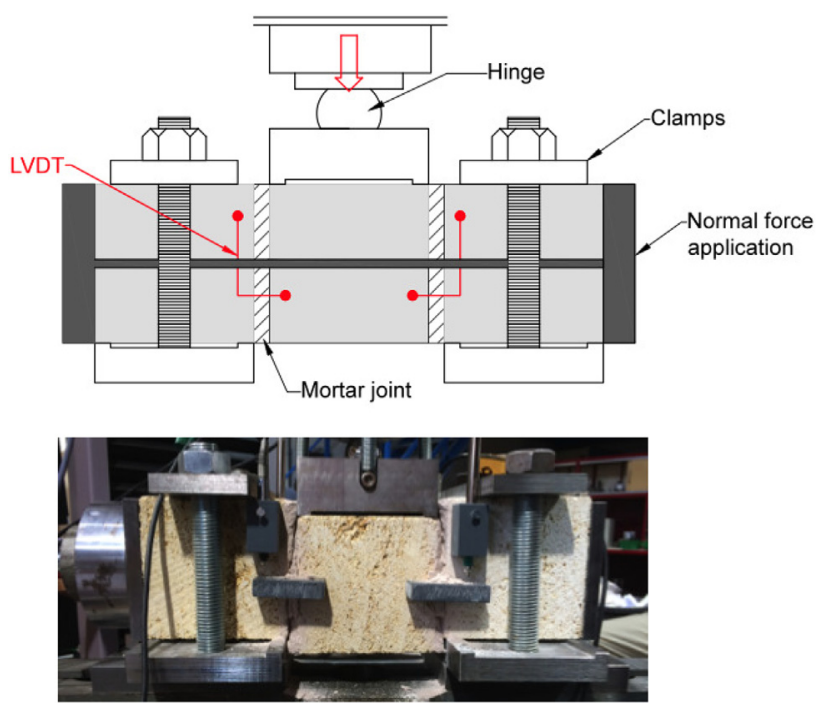

Fig. 9. Shear test set-up. 


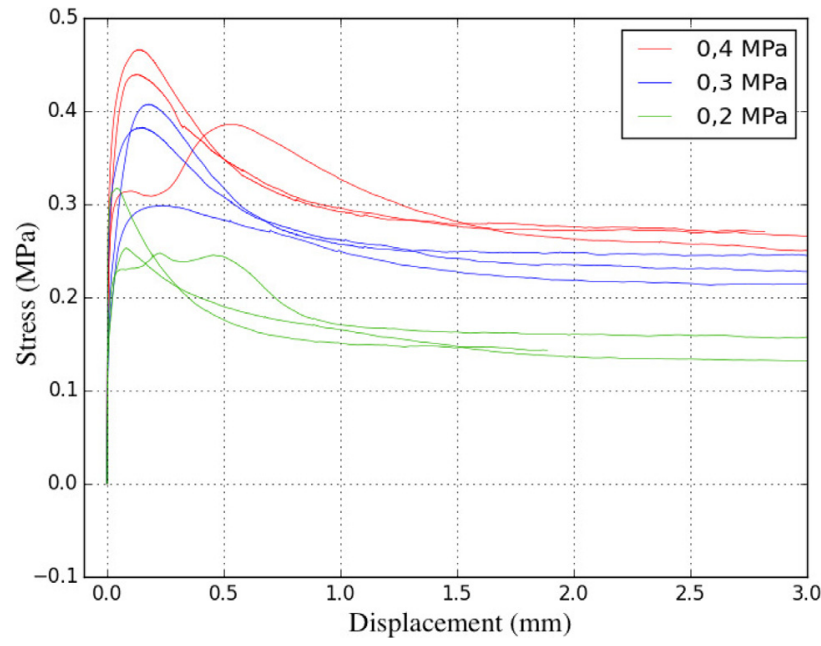

Fig. 10. Experimental curves from triplet shear tests.

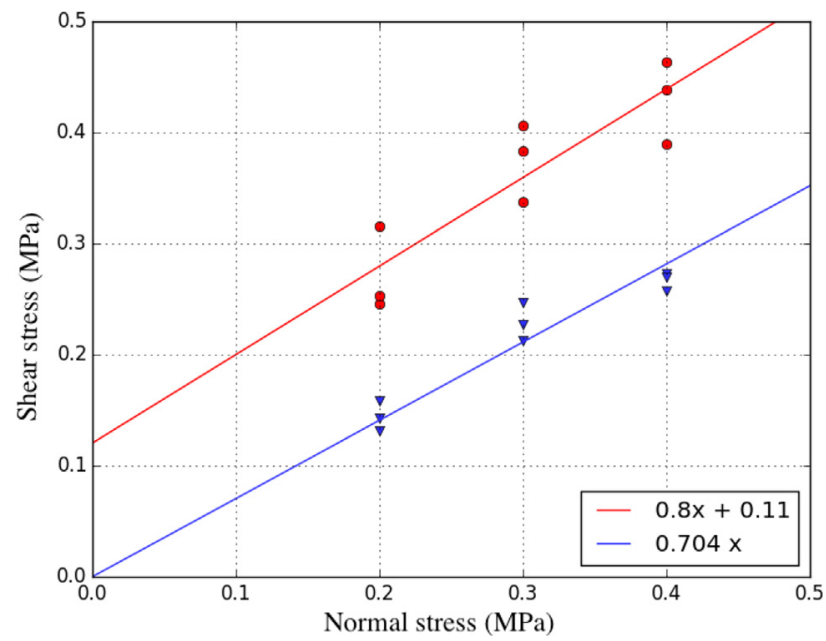

Fig. 11. Linear regressions for the determination of maximal shear stress (in red) and friction coefficient (in blue). (For interpretation of the references to colour in this figure legend, the reader is referred to the web version of this article.)

\subsubsection{Experimental results}

The stress-displacement responses obtained from the shear tests are plotted in the Fig. 10 according to the three levels of normal stress: 0.2, 0.3 and $0.4 \mathrm{MPa}$. Shear stress is defined as the vertical force divided by the surface of the two joints. Note that joint surface decreases during

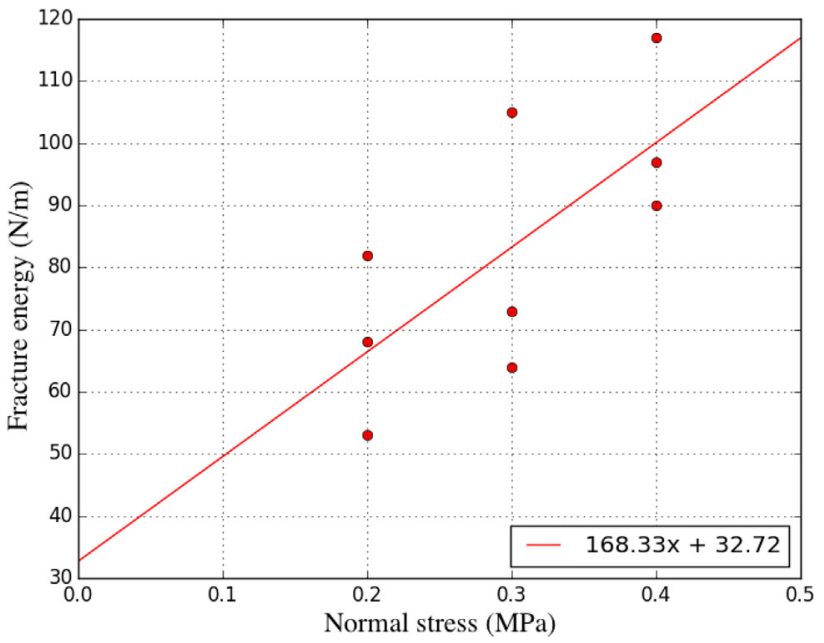

Fig. 13. Evolution of Mode II cohesive energy as function of normal compressive stress.

Table 2

Cohesive parameters obtained in Mode II.

\begin{tabular}{cc}
\hline \multicolumn{3}{c}{ Mode II } \\
\hline$\sigma_{I I}^{e}(\mathrm{MPa})$ & $0.11(0.09-0.14)$ \\
$K_{I I}^{0}(\mathrm{~Pa} / \mathrm{m})$ & $5.8 \times 10^{10}(0.14-0.39)$ \\
$J_{I I}^{c}\left(\mathrm{~J} / \mathrm{m}^{2}\right)$ & $34(0.14-1.21)$ \\
$\delta_{I I}^{U}(\mathrm{~mm})$ & $2.51(0.10-0.23)$ \\
\hline
\end{tabular}

the test due to the shear displacement. Consequently, the shear stress is continuously computed from the corresponding actual joint surface, as well as the normal stress, bit this latter remains approximately constant during the test. As shown in Fig. 10, each stress-displacement response exhibits a residual stress for large values of the shear displacement, corresponding to the frictional behaviour of the failed joint.

As expected from the Coulomb's law, the value of the residual frictional stress increases as a function of the normal stress level. The linear regression of these residual stresses as function of normal stresses (with y-intercept forced to zero) leads to the estimation of the friction coefficient $\mu$ as shown from Fig. 11. Here, $\mu$ is estimated equal to 0.70 .

Moreover, from the direct observation of the frictional (residual) stress a decomposition of stress-displacement behaviour in terms of frictional and cohesive behaviours is proposed. As shown from Fig. 12, this decomposition consists in estimating the cohesive behaviour of the joint by subtracting the frictional stress from the observed stress-displacement response.
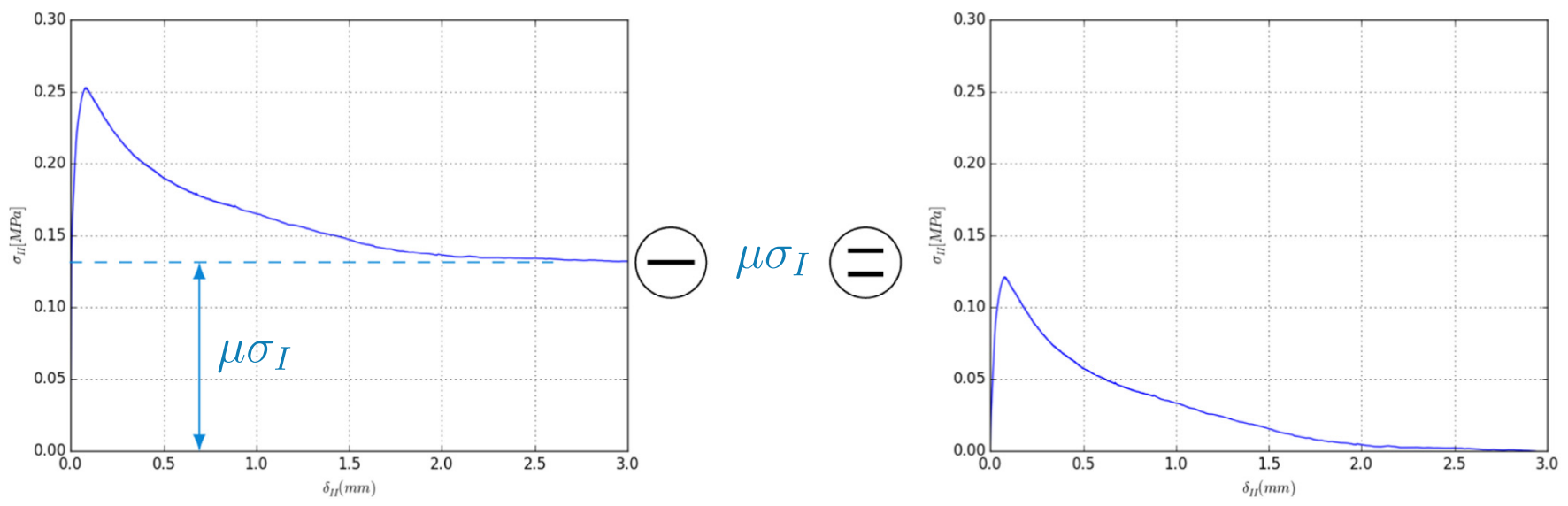

Fig. 12. Estimation of the cohesive behaviour of the joint from the subtraction of the residual stress linked to the frictional behaviour. 


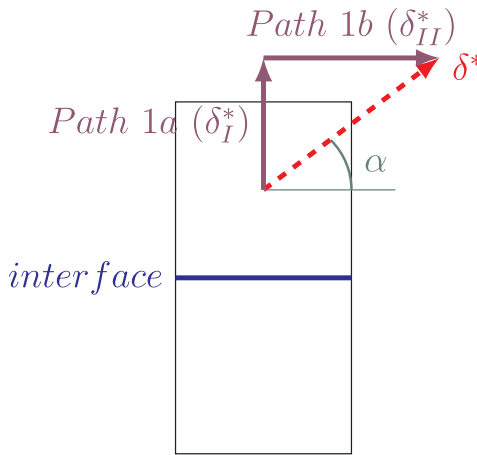

(a) Path 1

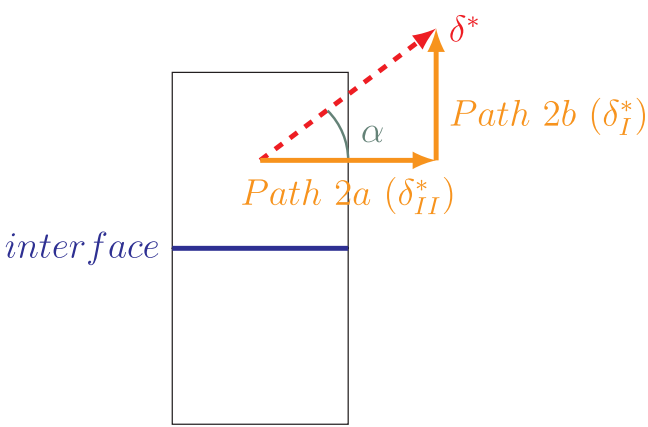

(b) Path 2

Fig. 14. Tested loading paths.

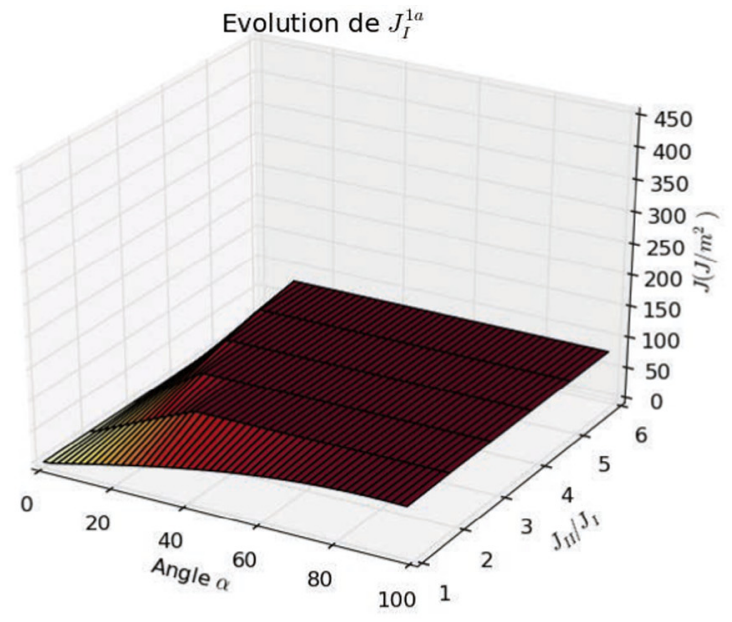

(a) Dissipated energy during Path 1a

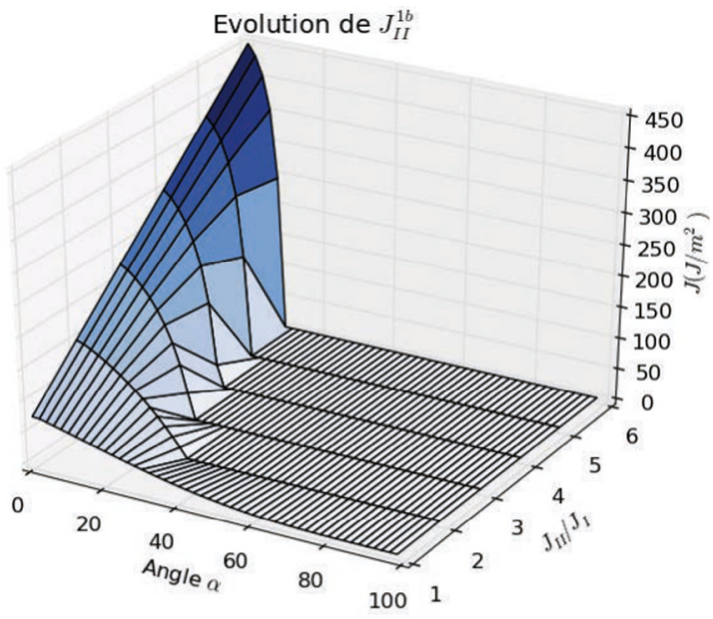

(b) Dissipated energy during Path $1 \mathrm{~b}$

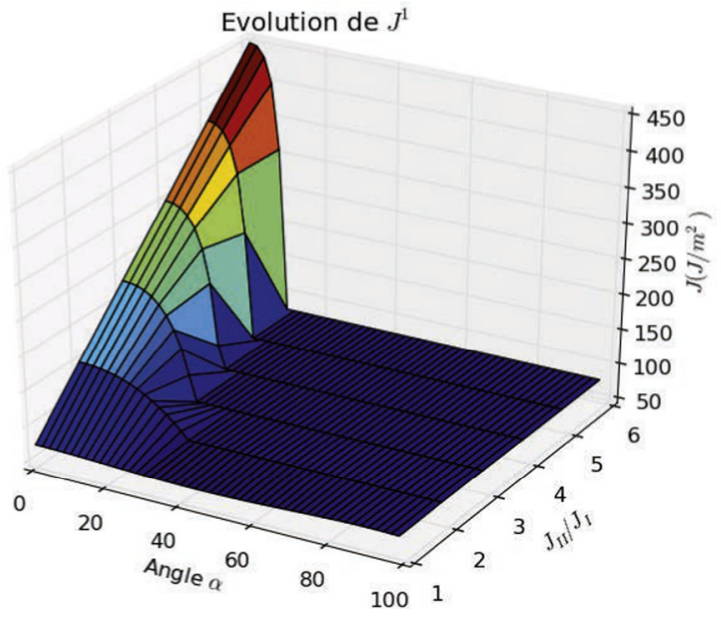

(c) Total dissipated energy during Path 1

Fig. 15. Influence of ratio $J_{I I} / J_{I}$ on the load path 1 dependancy.

Once the frictional behaviour is isolated, the cohesive parameters are estimated from an approach analogous to the one proposed for Mode I:

- The initial stiffness is obtained with a linear regression before the peak stress, when the response of the two joints is similar

- Cohesive fracture energy is defined as the area under the stressdisplacement curve, after subtracting the plateau due to friction (Fig. 12).
- Ultimate displacement is determined when the stress gets stabilised

The evolution of the maximal shear stress (i.e. $\sigma_{I I}^{e}+\mu \sigma_{I}$ ) and of the cohesive fracture energy against normal stress level are plotted in Figs. 11 and 13 respectively. The linear regression of the maximal shear stress shown in Fig. 11 exhibits a value of the slope quite closed to the value of the friction coefficient, which emphasises a quasi non-dependence of the maximal cohesive shear stress $\sigma_{I I}^{e}$ upon normal stress level. The y-intercept is assumed to be the maximal shear stress for zero 


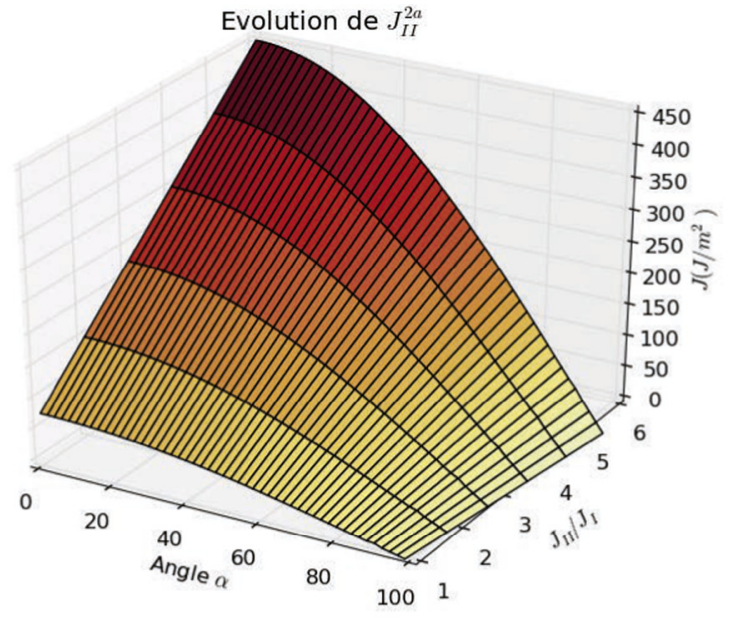

(a) Dissipated energy during Path 2a

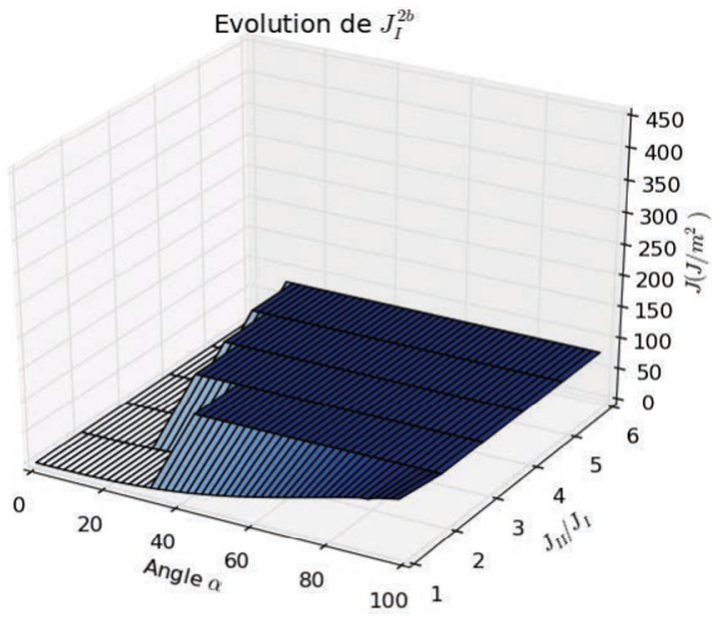

(b) Dissipated energy during Path $2 \mathrm{~b}$

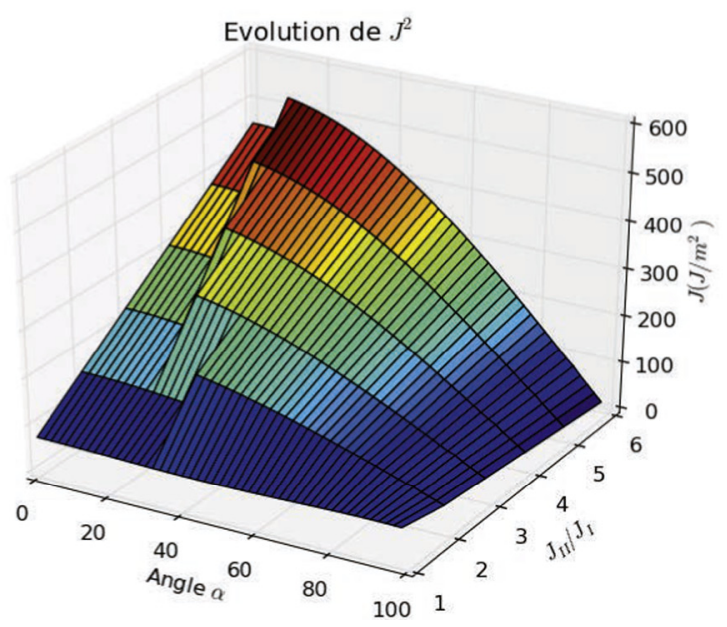

(c) Total dissipated energy during Path 2

Fig. 16. Influence of ratio $J_{I I} / J_{I}$ on the load path 2 dependancy.

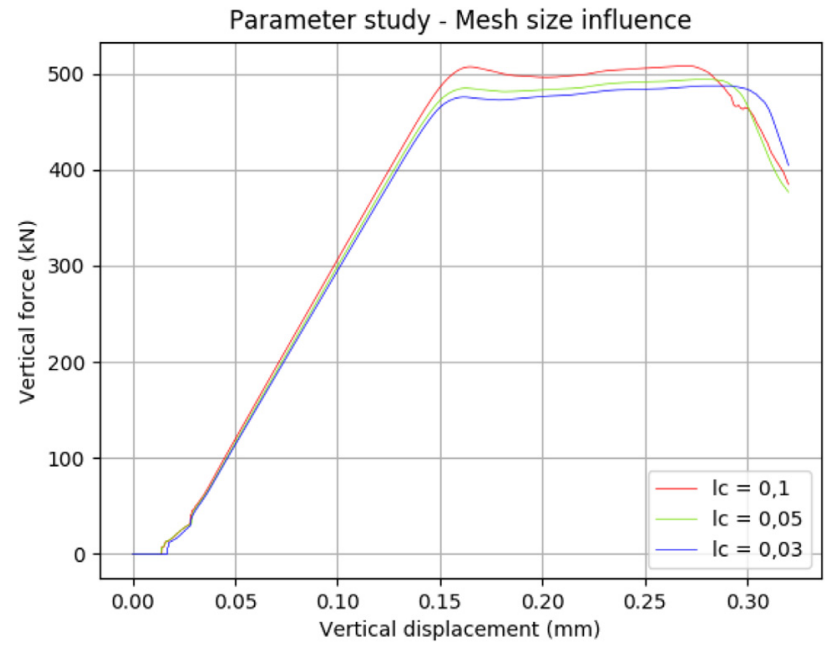

Fig. 17. Mesh size influence.

compression level.

On the other hand, as shown from Fig. 13, the Mode II cohesive fracture energy seems to be dependent on the normal stress. Even if such an observation was also made by Van der Pluijm [23], the few results and their large scattering do not allow to clearly conclude about this dependence. The observed normal stress dependent character of the Mode II cohesive energy could be induced by the arbitrary separation of the cohesive and frictional behaviours proposed here. Both behaviours could be coupled and their separation be more complex than a simple subtraction. Here, the cohesive energy for zero normal compression stress will be taken as the $y$-intercept of the linear regression of cohesive energies as function of normal stresses.

Table 2 summarises the cohesive parameters obtained for Mode II for the given assembly. An interval is given for the coefficient of variation since they were computed for each stress level.

\section{Mixed mode modelling of masonry}

\subsection{Path-dependency of mixed-mode formulation on a basic sample}

The aforementioned formulation is path-dependent, meaning that fracture energy will be consumed differently as function of the loading path. This kind of behaviour was already noticed with other models, especially the one proposed by Snozzi and Molinari [31] formulated for masonry mortar joints. Such a dependence seems in agreement with quasi-brittle fracture behaviour for which cracks develop in directions perpendicular to the extension of material.

To highlight this load-dependency, we study analytically two blocks, loaded with two different load paths that have the same 


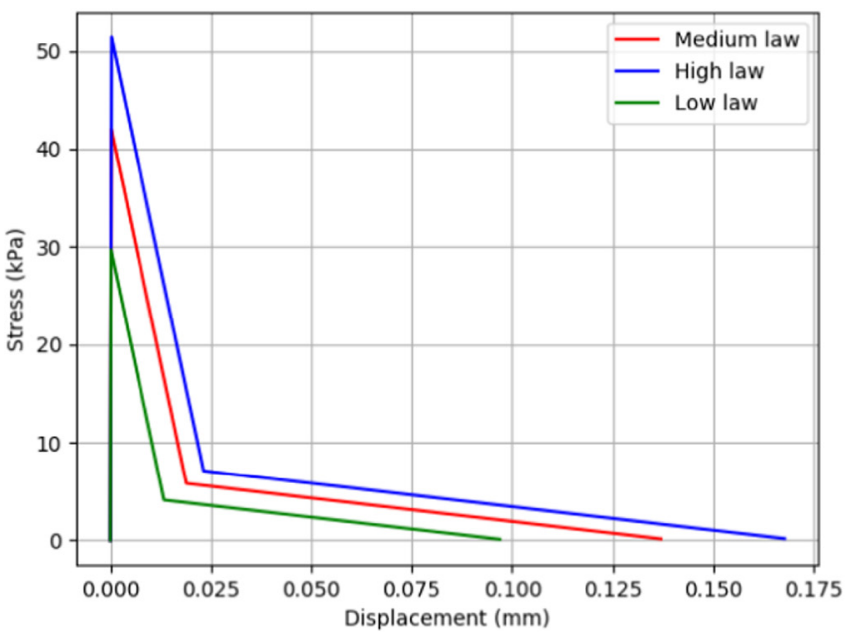

(a) Mode I laws

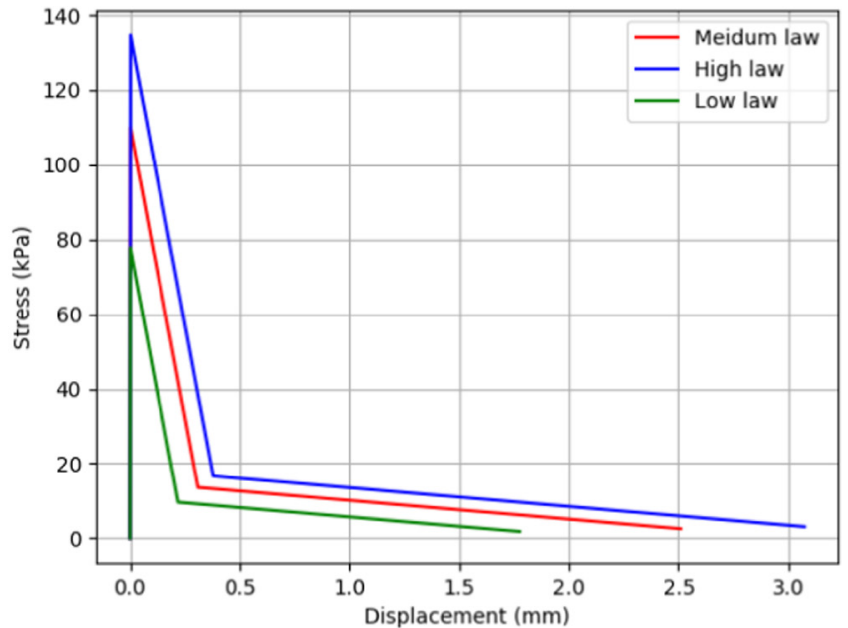

(b) Mode II laws

Fig. 18. Mode I and Mode II laws used for parametric study.

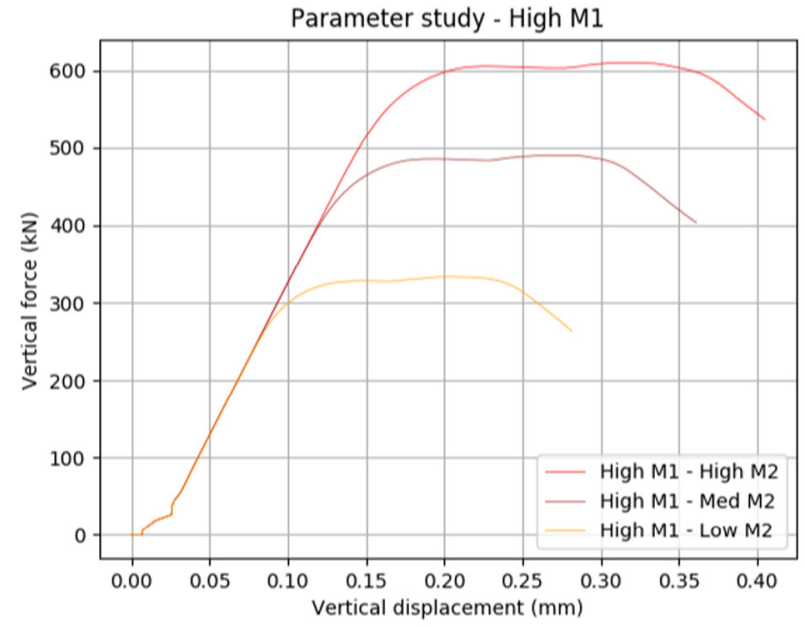

(a) High Mode I combinations

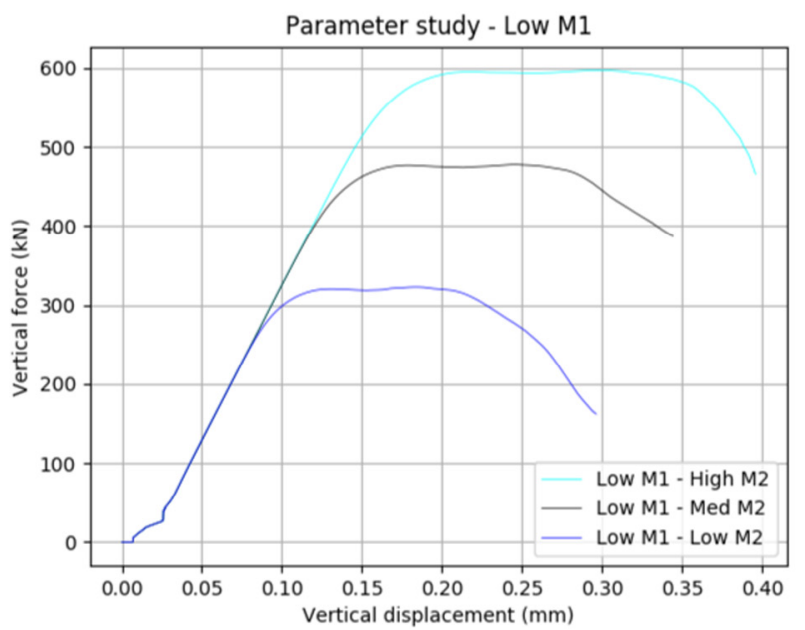

(c) Low Mode I combinations

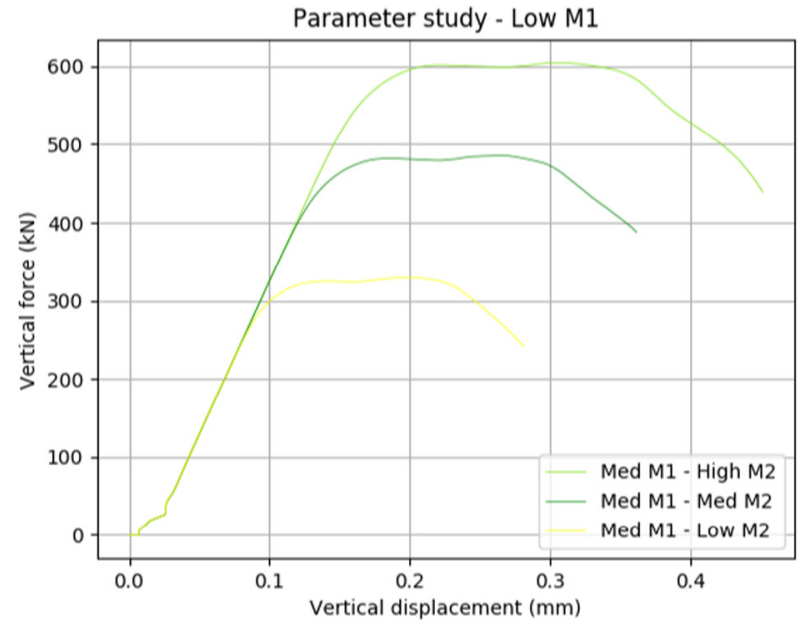

(b) Medium Mode I combinations

Fig. 19. Load-displacement responses obtained from simulation of diagonal compression tests.

departure and arrival points (Fig. 14). The dissipated cohesive energy is expressed and compared for the two studied paths.

In the Path 1, the interface is first loaded with pure traction until $\delta_{I}^{*}$ displacement (Path 1a), then in pure shear until $\delta_{I I}^{*}$ (Path 1b) as shown in Fig. 14 a. During Path 1a, the interface works in Mode I and the energy dissipated until $\delta_{I}^{*}$ can be written as:

$J_{I}^{1 a}=\frac{1}{2} \sigma_{I}^{e} \delta_{I}^{*} d^{1 a}$ 


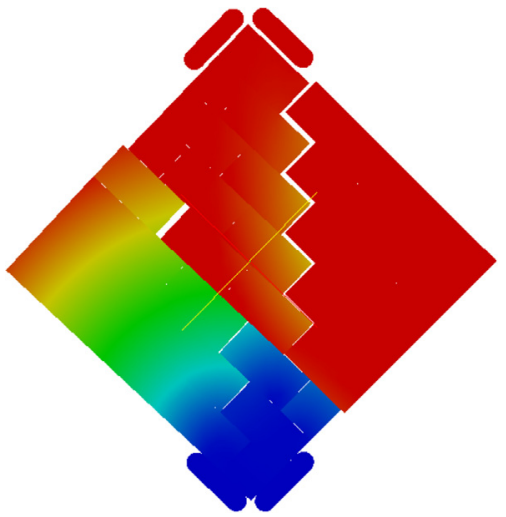

Fig. 20. Typical failure mode.

where $d^{1 a}$ corresponds to the value of the damage variable at the end of Path 1a.

Then, shear loading (corresponding to Path $1 \mathrm{~b}$ ) is applied until $\delta_{I I}^{*}$. The dissipated energy corresponding to this second path can be expressed as:

$J_{I I}^{1 b}=\frac{1}{2} K_{0} \delta_{I I}^{1 a} \delta_{I I}^{*}\left(d^{1 b}-d^{1 a}\right)$

where $d^{1 b}$ is the value of the damage variable at the end of the Path $1 \mathrm{~b}$.

According to Eqs. (18) and (19), the total dissipated energy during Path 1 (i.e. $J_{I}^{1 a}+J_{I I}^{1 b}$ ) can be computed as a function of the loading angle $\alpha$ and of the ratio $J_{I I}^{c} / J_{I}^{c}$ ranging from 1 to 6 . The obtained surface is plotted in Fig. 15. It appears that for a ratio $J_{I I} / J_{I}$ equal to one, the total dissipated energy is the same regardless from the loading angle. However, the more the ratio $J_{I I}^{c} / J_{I}^{c}$ is important, the more dissipated energy increases for lower values of $\alpha$. For $\alpha$ greater than 30 degrees, there is no more difference in dissipated energy because the interface fails in Mode I for which cohesive energy is smaller than the one of Mode II.
According to the Path 2 plotted in Fig. 14b, the interface is first loaded with pure shear until $\delta_{I I}^{*}$ displacement (Path 2a), then in pure traction until $\delta_{I}^{*}$ (Path $2 \mathrm{~b}$ ). The energy dissipated during Path 2a (i.e. until $\delta_{I I}^{*}$ ) can be written as:

$J_{I I}^{2 a}=\frac{1}{2} \sigma_{I I}^{e} \delta_{I I}^{*} d^{2 a}$

Then, the dissipated energy relative to Path $2 \mathrm{~b}$ leads to:

$J_{I}^{2 b}=\frac{1}{2} K_{0} \delta_{I}^{2 a} \delta_{I}^{*}\left(d^{2 b}-d^{2 a}\right)$

where $d^{2 a}$ and $d^{2 b}$ correspond to the values of the damage variables after loading paths $2 \mathrm{a}$ and $2 \mathrm{~b}$ respectively.

The total dissipated energy during Path 2 (i.e. $J_{I I}^{2 a}+J_{I}^{2 b}$ ) is plotted in the Fig. 16 as function of the loading angle $\alpha$ and of the ratio $J_{I I} / J_{I}$. The comparison of the dissipated energies during Path 1 (Fig. 15) and Path 2 (Fig. 16) exhibits a strong path dependancy, especially for large values of the ratio $J_{I I}^{c} / J_{I}^{c}$.

\subsection{Analysis of the diagonal compression on a masonry panel}

The ability of the mixed mode contact law to describe masonry failure modes is tested from the modelling of a diagonal compression test on a masonry. The test conditions correspond to those of the ASTM standard test method used to estimate the tensile or shear strength of masonry [3]. The test is performed by applying a compression force in the diagonal direction of a squared masonry panel until failure. The setup induce a complex stress state at the center of the panel. When mortar strength is sufficiently strong, the failure occurs experimentally from crack opening located at the center of the panel, which propagates from the center to the corners of the panel in its diagonal direction [1]. The specimen tends to split apart with a crack in the direction parallel to the load axis.

LMGC90 software is used for the modelling, considering deformable blocks which are extended to take into account mortar joint thickness.

\section{MODE II}

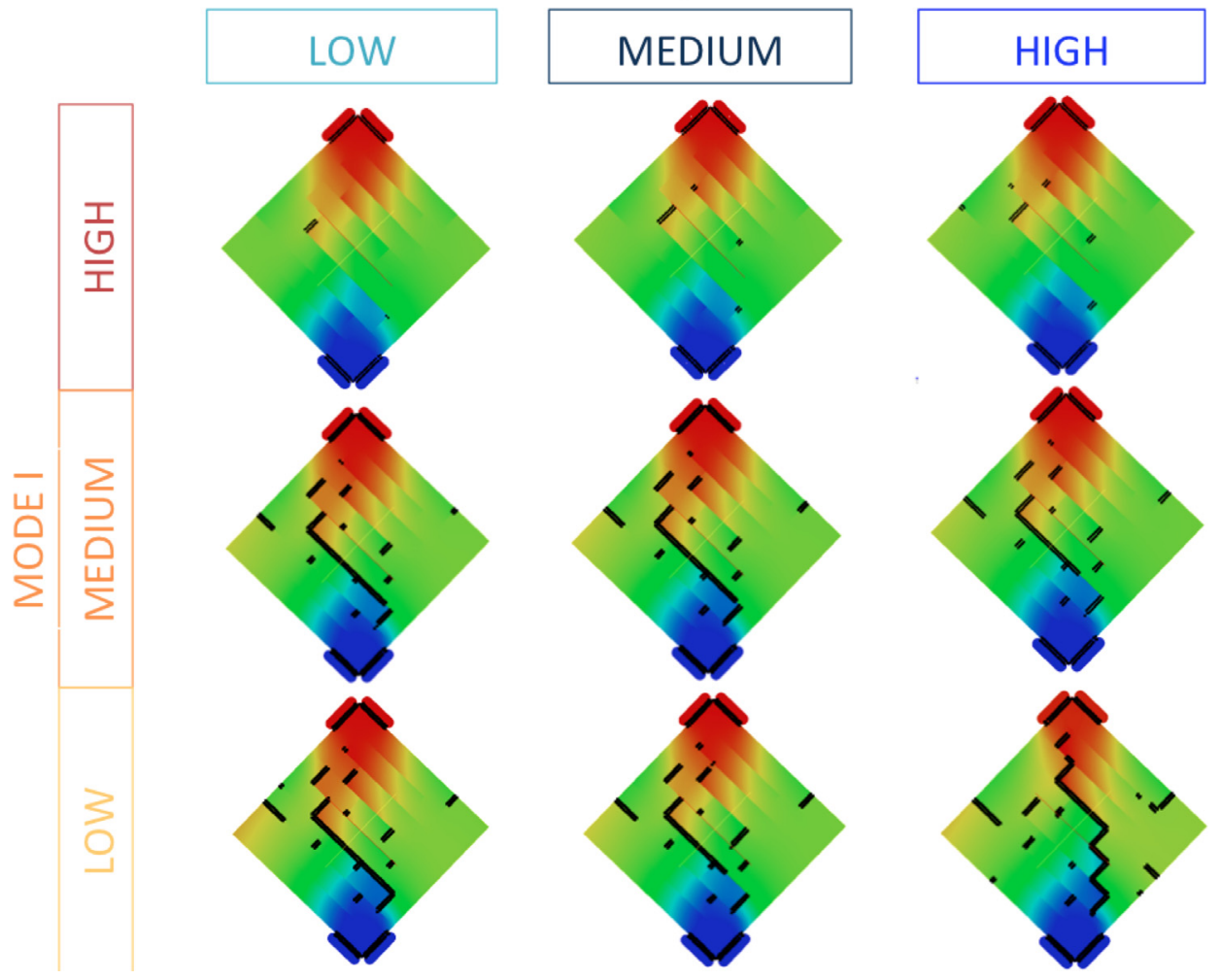

Fig. 21. Broken contacts (i.e., cracks) just before panel failure. 


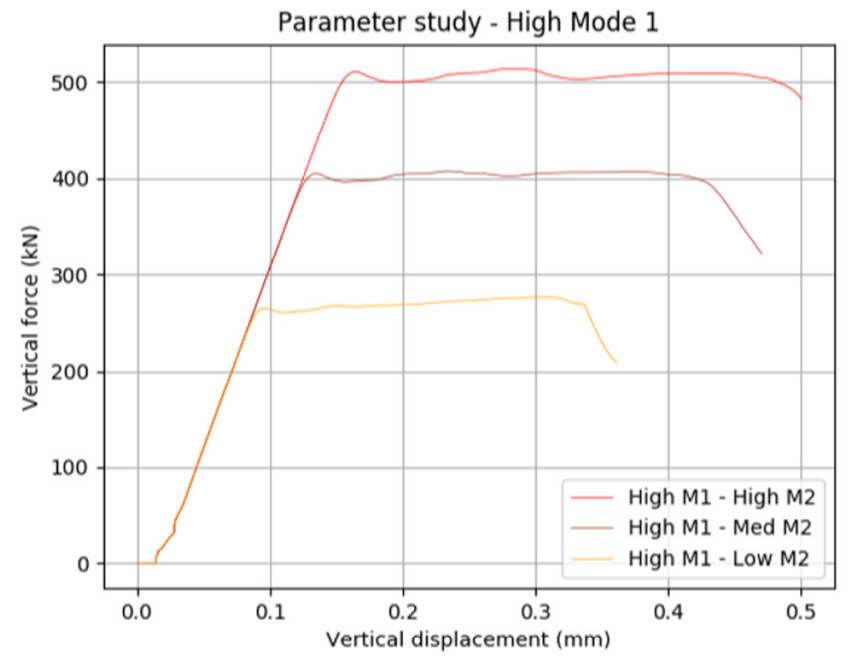

(a) High Mode I combinations

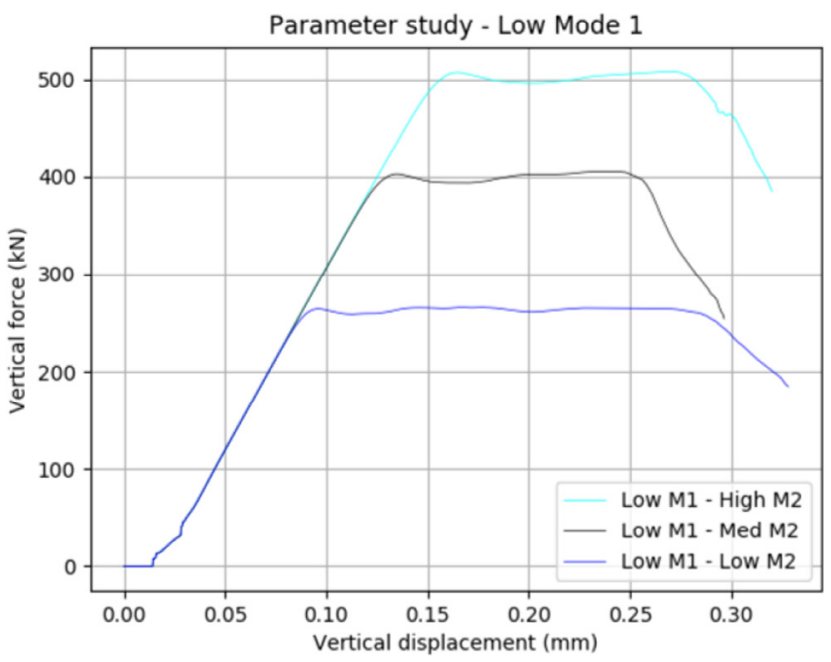

(c) Low Mode I combinations

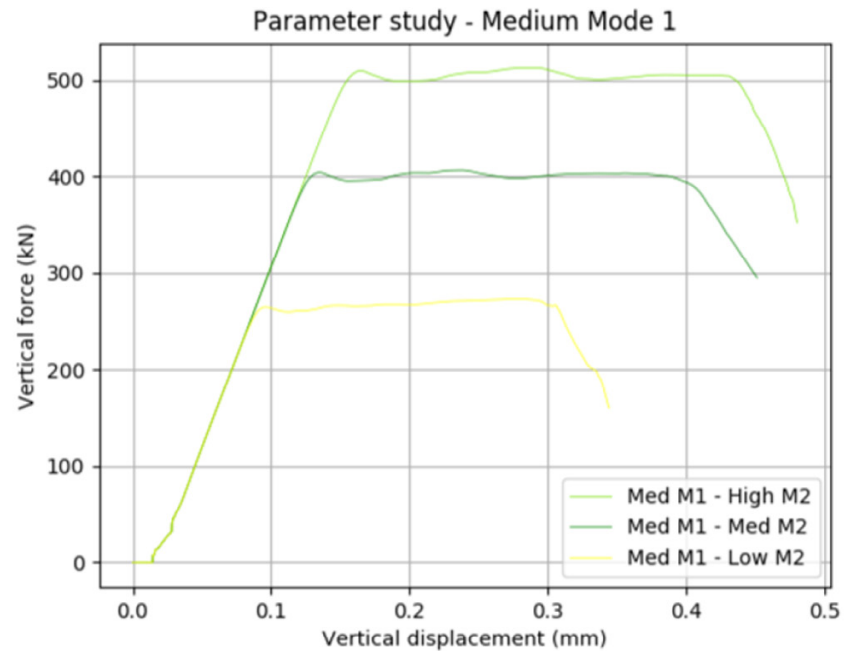

(b) Medium Mode I combinations

Fig. 22. Load-displacement responses obtained from simulation of diagonal compression tests (panel compounded from half bricks).

The Young modulus of extended blocks is thus adapted to be representative of the stone/mortar assembly. The panel is placed with its diagonal perpendicular to the ground. The lower corner is fixed while an increasing displacement is applied at the upper corner in the diagonal direction. The cohesive contact law exposed above is used to represent mortar joints behaviour at the interface between blocks.

\subsubsection{Influence of the mesh size}

Firstly, the influence of the mesh size on the load-displacement response is studied. The size of the mesh is controlled by the parameter $l_{c}$. This parameter defines the number of finite elements along each sides of a block $N_{E F / \text { side }}$ such as $N_{E F / \text { side }}=1 / l_{c}$. Thus, the smaller $l_{c}$ is, the more refined the mesh is and the more the contact points between blocks are since there is two contact points by finite element edge. Five sizes of meshing are considered corresponding to $l_{c}$ values equal to $0.5,0.2,0.1,0.05$ and 0.03 . Numerical simulations are carried out from each mesh considering the cohesive laws of Mode I and Mode II described from the cohesive parameters given in Tables 1 and 2 respectively. The load-displacement response obtained for each mesh are plotted in Fig. 17. The different load-displacement curves (whose shape will be discussed in the following) converge towards the same response for values of $l_{c} \leqslant 0.1$ and hence, $l_{c}=0.1$ is considered in the following of the study.

\subsubsection{Influence of cohesive parameters}

In a second step, a parametric study is led to investigate the influence of input data on numerical results. In this parametric study, the cohesive laws of Mode I and Mode II defined from the cohesive parameters given in Tables 1 and 2 (obtained from experiments) are considered as the reference laws and will be named as medium laws in the following. We also consider low Mode I and Mode II laws (resp. high Mode I and Mode II laws), proportional to medium laws, corresponding to cohesive energies and maximum stress decrease (resp. increase) by $50 \%$ while the initial stiffnesses $K_{I}^{0}$ and $K_{I I}^{0}$ are kept constant. Low, medium and high Mode I and Mode II laws are plotted in Fig. 18.

We study combinations of the three laws of each mode which gives us 9 input sets. Load-displacement responses of the panel obtained for each combination are plotted in Fig. 19. All curves exhibit an elastic part, which is the same regardless from the input data, followed by a plateau at constant load before failure. The load value of the plateau as well as the extent of the plateau (in terms of displacement) increase as a function of the level of Mode II law while the level of the Mode I law does not seem to influence the response. Thus, the more cohesive energy and maximum stress of Mode II increase, the more the load value and the extent of the plateau are higher.

Note that, if the tests were carried out using load control instead of displacement one, the load-displacement response of the panel would 


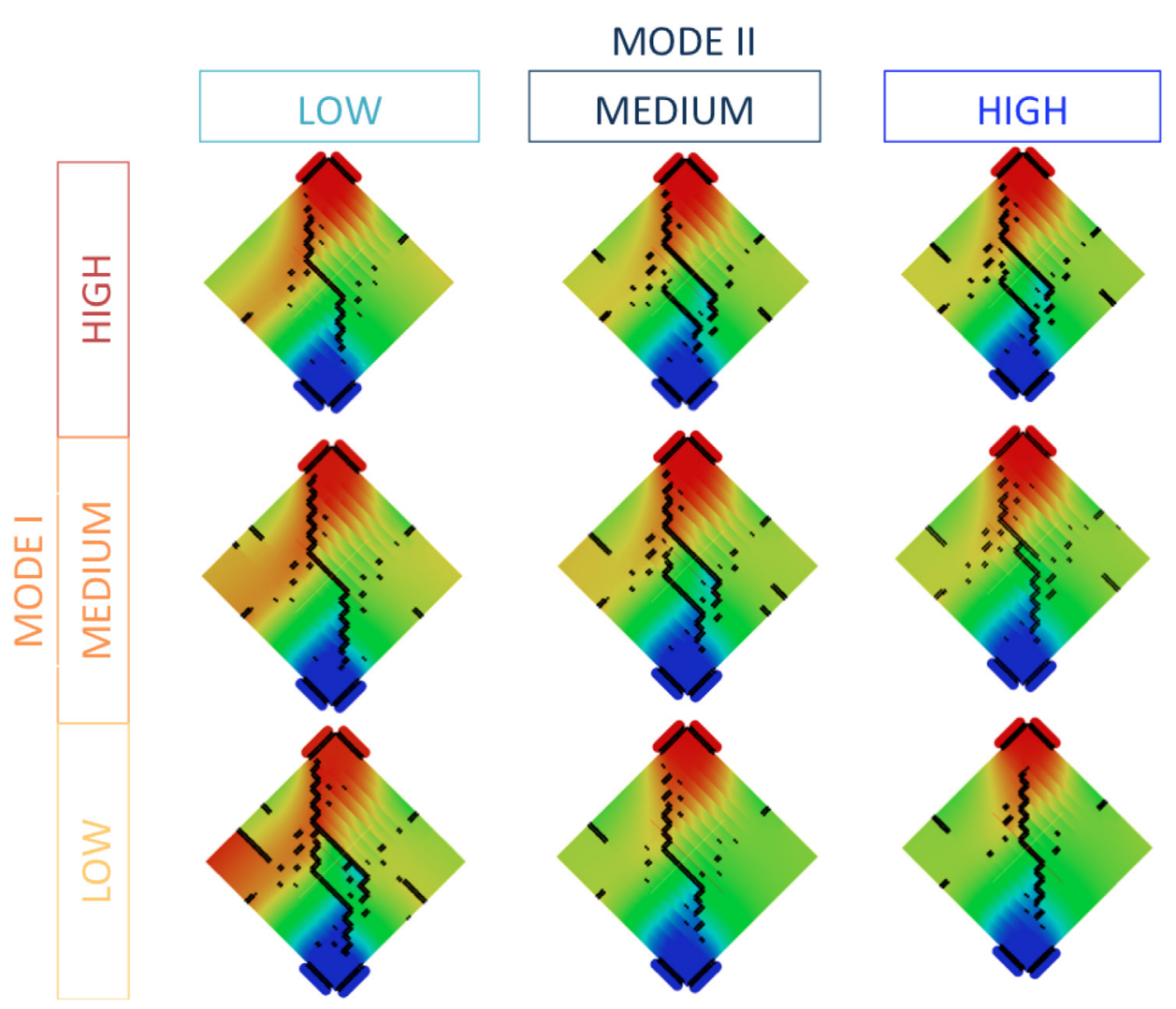

Fig. 23. Broken contacts (i.e., cracks) just before panel failure (panel compouned of half bricks).

Table 3

Mechanical properties of masonry and constituents extracted from Gabor et al. [11].

\begin{tabular}{lcc}
\hline & Bricks & Mortar \\
\hline Elastic modulus (GPa) & 12.8 & 4.0 \\
Shear strength (MPa) & - & 1.63 \\
Residual friction angle & - & $43^{\circ}$ \\
\hline
\end{tabular}

have been truncated at the onset of the load plateau and hence could be qualified as a brittle response. Moreover, (i) the peak load being mainly dependent on the Mode II cohesive properties (as previously mentioned) and (ii) the load-displacement response being few non-linear before the peak load (emphasizing a low damage level of the mortar joint), it is expected that the peak load magnitude is mainly governed by the maximal cohesive shear stress $\sigma_{I I}^{e}$.

On the other hand, a typical failure obtained for the medium Mode I and Mode II laws is shown in Fig. 20. As experimentally expected, a diagonal crack appears and the panel splits apart. On this basis, the final failure modes obtained from each laws combination did not exhibit significant difference compared to the one shown in Fig. 20 and hence are not plotted in the paper. Nevertheless, if difference between final failure modes are not significant, the location of damage at the end of the plateau of the load, i.e., at the onset of the whole failure exhibits differences as a function of the considered combination. Indeed, as shown from Fig. 21 in which the broken contacts (black dots) are plotted at the end of the plateau of the load, the number of broken contacts and hence of cracks are greater for combination using the lower Mode I law.

In a last step, let us investigate the influence of the size of the blocks on the mechanical response of the panel in studying a panel of the same dimensions but with blocks twice as small. For these simulations, the nine combinations of Mode I and Mode II laws previously used lead to the load-displacement responses plotted in Fig. 22. Compared to responses of full-bricks panels shown in Fig. 19, the load-displacement curves plotted in Fig. 22 exhibit an analogous shape of response but characterised by a longer plateau and values of plateau load lower than those observed for full bricks panels. As observed for full bricks panels, the magnitude of the load-displacement responses is mainly governed by the level of the Mode II law while the influence of mode I is not significant on the panel response.

Finally, the location of damage at the end of the plateau load (i.e., just before the panel failure) shown in Fig. 23 does not exhibit significant difference as a function of the combination of the Mode I and mode II laws. However, one can note the main crack is more localized when the difference between pure modes levels increases.

\subsubsection{Comparison of experimental and simulated responses of masonry panel}

In this last part, a comparison of experimental and simulated responses of a masonry panel under diagonal compression is proposed. Among different experimental studies available in literature $[5,11,17]$, the one relative to the work of Gabor et al. [11] is chosen for the quality of the experimental responses obtained. Gabor et al. [11] tested two masonry panels (dimensions of $870 \times 840 \times 100 \mathrm{~mm}^{3}$ ) composed of hollow bricks $\left(210 \times 50 \times 100 \mathrm{~mm}^{3}\right)$ and $10 \mathrm{~mm}$ thick mortar joint (ready-to-use mortar with a $0-5 \mathrm{~mm}$ sand and Portland cement composition). The mechanical properties of masonry constituents used by Gabor et al. [11] are reported in Table 3.

The diagonal compression load was applied gradually on the corners of the panel and controlled by a load cell while the diagonal displacement of the masonry panel was measured by LVDT transducers. The experimental load-diagonal strain responses obtained by Gabor et al. [11] are reported in Fig. 24(a) (blue and green curves only, the red one having been obtained by Gabor et al. [11] from an EF simulation).

LMGC90 software is used again for the modelling, considering deformable blocks which are extended to take into account mortar joint thickness. Note that a slight modification of the panel dimensions are 


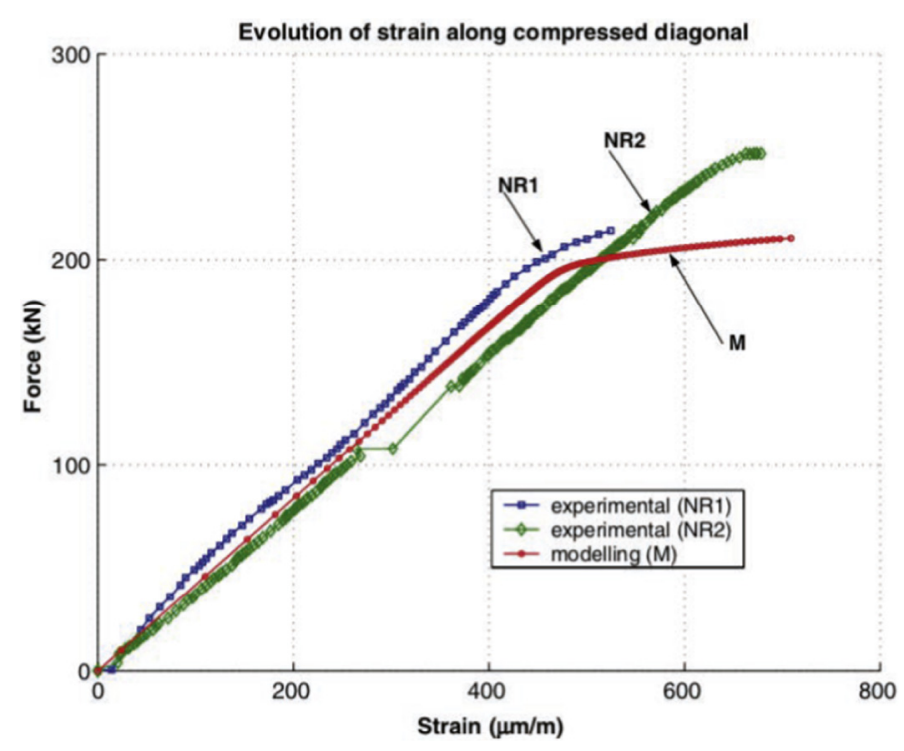

(a)

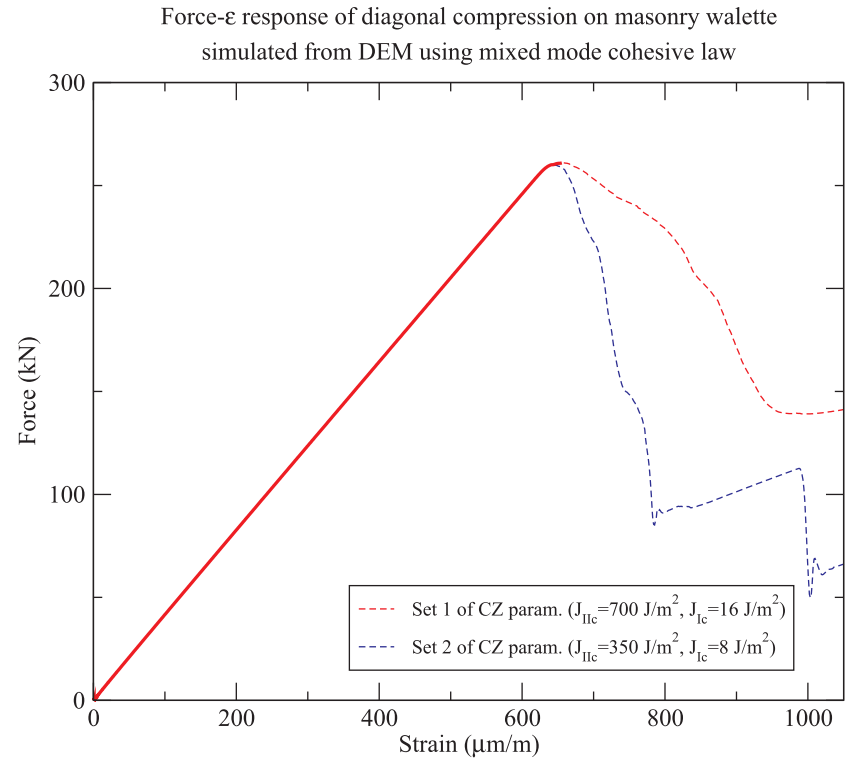

(b)

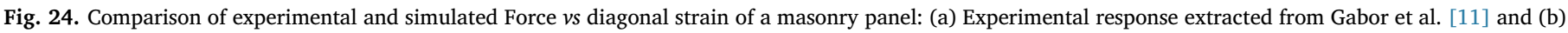
Simulated response obtained from DEM using mixed-mode cohesive law.

Table 4

Mechanical properties and cohesive zone parameters used in DEM simulations.

\begin{tabular}{|c|c|c|c|c|}
\hline & & \multicolumn{2}{|l|}{ Set 1} & Set 2 \\
\hline \multicolumn{5}{|c|}{ Cohesive zone parameters } \\
\hline \multirow[t]{4}{*}{ Mode II } & $\sigma_{I I}^{e}(\mathrm{MPa})$ & 1.63 & & 1.63 \\
\hline & $J_{I I}^{c}\left(\mathrm{~J} / \mathrm{m}^{2}\right)$ & 700 & & 350 \\
\hline & $\delta_{I I}^{u}(\mathrm{~mm})$ & 4.0 & & 4.0 \\
\hline & $\Phi_{I I}(1)$ & 0.5 & & 0.5 \\
\hline \multirow[t]{4}{*}{ Mode I } & $\sigma_{I}^{e}(\mathrm{MPa})$ & 0.33 & & 0.33 \\
\hline & $J_{I}^{c}\left(\mathrm{~J} / \mathrm{m}^{2}\right)$ & 16 & & 8 \\
\hline & $\delta_{I}^{u}(\mathrm{~mm})$ & 1.0 & & 1.0 \\
\hline & $\Phi_{I}(1)$ & 0.5 & & 0.5 \\
\hline \multicolumn{3}{|c|}{ Friction coefficient (1) } & 0.8 & \\
\hline \multicolumn{5}{|c|}{ Mechanical properties of extended block } \\
\hline \multicolumn{2}{|c|}{ Elastic modulus (GPa) } & & 8.26 & \\
\hline \multicolumn{2}{|c|}{ Poisson's ratio } & & 0.2 & \\
\hline \multicolumn{2}{|c|}{ Density $\left(\mathrm{kg} / \mathrm{m}^{3}\right)$} & & 1200 & \\
\hline
\end{tabular}

considered (dimensions of $840 \times 840 \times 100 \mathrm{~mm}^{3}$ ) since we used extended block of dimensions $210 \times 60 \times 100 \mathrm{~mm}^{3}$. The Young modulus of extended blocks is thus adapted to be representative of the stone/ mortar assembly and is given in Table 4 .

Concerning the Mode I and Mode II cohesive properties of the interface between extended blocks, only the maximal cohesive shear stress $\sigma_{I I}^{e}$ can be estimated from the shear strength of mortar (1.63 MPa) obtained by Gabor et al. [11] as reported in Table 3. In the absence of more informations about fracture properties of mortar joint, all other values of cohesive parameters are assumed with respect to the expected cohesive behaviours of a quasi-brittle material such as mortar and are given in Table 4. Nevertheless, as previously mentioned in Section 5.2 .2 , it is expected that the peak load magnitude is mainly governed by the maximal cohesive shear stress $\sigma_{I I}^{e}$ and hence, the other cohesive parameters are expected to be of less importance in this case. As such, note that two sets of cohesive parameters with the same value of the maximal cohesive shear stress $\sigma_{I I}^{e}=1.63 \mathrm{MPa}$ are considered (Table 4) for the numerical simulations in order to check this point. Thus, Mode I and Mode II cohesive energies of the set 1 of cohesive properties are considered twice as large as those of the set 2 (Table 4 ) while the initial stiffness of cohesive behaviours $K_{I I}^{0}$ and $K_{I}^{0}$ are considered here as penalty stiffness (i.e., $K_{i}^{0} \rightarrow \infty$ with $i=I, I I$ ).

As previously mentioned, the panel is placed with its diagonal perpendicular to the ground. The lower corner is fixed while an increasing displacement is applied at the upper corner in the diagonal direction. The Force-Diagonal strain responses of the panel obtained from the DEM simulations and corresponding to the two sets of cohesive parameters given in Table 4 are plotted in Fig. 24(b). Both ForceDiagonal strain responses are superimposed up to the peak load and differs in the post peak regime. As expected, the peak loads obtained from both sets of cohesive parameters are approximately the same (around $260 \mathrm{kN}$ ) and seems effectively linked to the value of the maximal cohesive shear stress $\sigma_{I I}^{e}$ while the load in post peak regime decreases more quickly in the case of weak Mode I and Mode II cohesive energies as expected intuitively.

Let's remember that the Force-Diagonal strain responses plotted in Fig. 24(b) are obtained from a displacement controlled test and must be truncated after the peak load in order to be compared to the responses expected from a load controlled test as performed by Gabor et al. [11]. On this basis, Fig. 24 shows that the simulated Force-Diagonal strain responses are in agreement with the experimental ones obtained by Gabor et al. [11] and this in terms of initial stiffness as well as in terms of peak load values.

\section{Conclusions}

A new contact law has been implemented in LMGC90 software allowing to take into account progressive damage of masonry mortar joints and their frictional post-failure behaviour. The proposed contact law is inspired from Cohesive Zone Models (with a bilinear approximation of the softening behaviour) and a frictional threshold is considered in Mode II in the case of normal compressive stress on the interface.

The mixed-mode formulation of CZM is based on pure modes behaviours and allows to consider any space configuration in order to be closer to actual mechanisms. Main advantage of the present model is that input parameters can be directly deduced from two experimental 
fracture tests especially designed for this purpose. A direct tensile test performed on two stone blocks bonded by one mortar joint allows for Mode I fracture characterization and a shear test carried on three stone blocks bonded by two mortar joints permits Mode II fracture and frictional characterization. The exploitation of experimental results is thus easy and input data of frictional cohesive law are quickly deduced.

First simulations of a masonry panel under diagonal compression has shown good agreement with experimental results in terms of failure modes and failure kinematics. Parametric study carried out on masonry panel under diagonal compression shows good agreement with experimental failure modes. As expected, input data influence response of the structure in terms of admissible load and damage propagation, but final failure modes stay globally unchanged. It is shown that the peak load value is strongly dependent on the Mode II cohesive parameters and especially the value of the maximal cohesive shear stress $\sigma_{I I}^{e}$. Finally, the simulation of the Load-Diagonal strain response of a masonry panel composed of hollow bricks and thick mortar joint exhibits a fair agreement with experimental responses and confirms that the value of peak load is effectively linked to the value of maximal cohesive shear stress $\sigma_{I I}^{e}$.

This contact law could be improved in studying the coupling between friction and cohesive behaviors. An accurate description of this coupling could avoid to consider Mode II cohesive energy depending on the normal stress level (as shown in Fig. 13) which is inconsistent from a theoretical point of view. Finally, if the softening behaviour of mortar in CZM is here described from a bilinear approximation, this one could be described from an exponential function which would describe the softening behaviour with a better accuracy.

\section{Acknowledgments}

The authors thank AIA Ingénierie (Architectes Ingénieurs Associés) company and the French National Research and Technology Agency (ANRT) for supporting the study. We also thank Bernard Solbes for his technical expertise in experimental tests. The experimental part of the study has been performed from the Xyloplate platform equipments Equipex XYLOFOREST (ANR-10-EQPX-16) - supported by the French National Research Agency (ANR).

\section{References}

[1] Alecci A, Fagone M, Rotunno T, De Stefano M. Shear strength of brick masonry walls assembled with different types of mortar. Construct Build Mater 2013;40:1038-45.

[2] Anthoine A. In-plane behaviour of masonry (a literature review). EUR, Luxembourg; 1992.

[3] ASTM E510-02. Standard Test Method for Diagonal Tension (Shear) in Masonry Assemblages. American Society for Testing Material; 2003.

[4] Barros J, Almeida J, Lourenco PB. Characterization of brick and brick-mortar interface under uniaxial tension. In: VII international seminar on structural masonry for developing countries; 2002. p. 67-76.

[5] Borri A, Castori G, Corradi M. Determination of shear strength of masonry panels through different tests. Int J Archit Heritage 2015;9:913-27.

[6] Casolo S, Milani G. Simplified out-of-plane modelling of three-leaf masonry walls accounting for the material texture. Constr Build Mater 2013;40:330-51.
[7] Cundall PA. Formulation of a three-dimensional distinct element model - Part I. A Scheme to detect and represent contacts in a system composed of many polyhedral blocks. Int J Rocks Mech Min Sci Geomech Abstracts 1988;25(3):107-16.

[8] Dubois F, Jean M, Renouf M, Mozul R, Martin A, Bagneris M. LMGC90. 10e Colloque National en Calcul des Structures; 2011.

[9] Dubois F, Renouf M, Jean M. Extensions d'une méthode par éléments discrets pour la modélisation de milieux divisés complexes. 8e Colloque National en Calcul des Structures, Giens; 2008.

[10] Dubois F, Acary V, Jean M. The contact dynamics method: a nonsmooth story. Comptes Rendus Mécanique 2018;346:247-62.

[11] Gabor A, Bennani A, Jacquelin E, Lebon F. Modelling approaches of the in-plane shear behaviour of unreinforced and FRP strengthened masonry panels. Compos Struct 2006;74:277-88.

[12] Gambarotta L, Lagomarsino S. Damage models for the seismic response of brick masonry shear walls. Part I: the mortar joint model and its applications. Earthq Eng Struct Dynam 1997;26(4):423-39.

[13] Hordijk DA. Local approach to fatigue of concrete. TU Delft, Delft University of Technology; 1991.

[14] Jean M. The non-smooth contact dynamics method. Comput Meth Appl Mech Eng 1999:235-57.

[15] Lotfi HR, Shing PB. An appraisal of smeared crack models for masonry shear wall analysis. Comput Struct 1991;41:413-25.

[16] Lourenço PB. Computational strategies for masonry structures. Ph.D. thesis, Technical University Delft, Delft University Press, The Netherlands, ISBN 90-4071221-2, February 1996; 1996.

[17] L. Malyszko, 2005. In-plane shear and tensile strength tests of small brickwork specimens. Structural Analysis of Historical Constructions, Modena, Lourenço and Roca (eds), 2005 Taylor \& Francis Group, London, ISBN 0415363799.

[18] Milani G, Lourenço P, Tralli A. 3D homogenized limit analysis of masonry buildings under horizontal loads. Eng Struct 2007;29(11):3134-48.

[19] Moreau J-J. Unilateral contact and dry friction in finite freedom dynamics. Nonsmooth Mechanics and Applications. Vienna: Springer; 1988. p. 1-82.

[20] Morel S, Lespine C, Coureau J-L, Planas J, Dourado N. Bilinear softening parameters and equivalent LEFM R-curve in quasi-brittle failure. Int J Solids Struct 2010;466:837-50.

[21] Page AW. An in-plane finite element model for brick masonry. ${ }^{* * *}$ Proc. Structural Congress 1985:1-18.

[22] Perales R. Modélisation du comportement mécanique par éléments discrets des ouvrages maçonnés tridimensionnels. Contribution à la définition d'éléments de contacts surfaciques. PhD Thesis, Université de Montpellier II; 2007.

[23] Van der Pluijm R. Out-of-plane bending of masonry behaviour and strength. PhD Thesis, Technische Universiteit Eindhoben; 1999.

[24] Rafiee A, Vinches M, Bohatier C. Modelling and analysis of the Nimes arena and the Arles aqueduct subjected to a seismic loading, using the Non-Smooth Contact Dynamics method. Eng Struct 2008;30:3457-67.

[25] Rekik A, Lebon F. Homogenization methods for interface modeling in damaged masonry. Adv Eng Software 2012;46(1):35-42.

[26] Rots JG. Numerical simulation of cracking in structural masonry. Heron, vol. 36, pp. 49-63.

[27] Sacco E, Toti J. Interface elements for the analysis of masonry structures. Int $\mathrm{J}$ Comput Methods Eng Sci Mech 2010;11(6):354-73.

[28] Shing PB, Klamerus E, Spaeh H, Noland JL. Seismic performance of reinforced masonry shear walls. Proceedings of the 9th world conference on earthquake engineering 1988;7:103-8.

[29] Silva LC, Lourenço PB, Milani G. Rigid block and spring homogenized model (HRBSM) for masonry subjected to impact and blast loading. Int J Impact Eng 2017;109:14-28.

[30] Silva LC, Lourenco PB, Milani G. Derivation of the out-of-plane behaviour of masonry through homogenization strategies: micro-scale level. Compos Struct 2018;209:30-43.

[31] Snozzi L, Molinari JF. A cohesive element model for mixed mode loading with frictional contact capability. Int J Numer Meth Eng 2013:510-26.

[32] Taforel P, Dubois F, Pagano S. Evaluation of numerical uncertainties on the modeling of dry masonry structures submitted to out-of-plane loading, using the NSCD method in comparison with experimental test. ECCOMAS 2012, Vienna, September $10-14 ; 2012$ 